San Jose State University

SJSU ScholarWorks

Master's Theses

Master's Theses and Graduate Research

Fall 2016

 Patterning Applications
}

Gabriela Alva

San Jose State University

Follow this and additional works at: https://scholarworks.sjsu.edu/etd_theses

\section{Recommended Citation}

Alva, Gabriela, "Directed Self-Assembly of High- $\chi$ Block Copolymers for Advanced Patterning Applications" (2016). Master's Theses. 4746.

DOI: https://doi.org/10.31979/etd.a629-gbrm

https://scholarworks.sjsu.edu/etd_theses/4746

This Thesis is brought to you for free and open access by the Master's Theses and Graduate Research at SJSU ScholarWorks. It has been accepted for inclusion in Master's Theses by an authorized administrator of SJSU ScholarWorks. For more information, please contact scholarworks@sjsu.edu. 


\title{
DIRECTED SELF-ASSEMBLY OF HIGH- $\chi$ BLOCK COPOLYMERS FOR ADVANCED PATTERNING APPLICATIONS
}

\author{
A Thesis \\ Presented to \\ The Faculty of the Department of Biomedical, Chemical, and Materials Engineering
}

San José State University

In Partial Fulfillment

of the Requirements for the Degree

Master of Science

by

Gabriela Alva

December 2016 
(C) 2016

Gabriela Alva

ALL RIGHTS RESERVED 
The Designated Thesis Committee Approves the Thesis Titled

DIRECTED SELF-ASSEMBLY OF HIGH- $\chi$ BLOCK COPOLYMERS FOR ADVANCED PATTERNING APPLICATIONS

By

Gabriela Alva

APPROVED FOR THE DEPARTMENT OF BIOMEDICAL, CHEMICAL, AND MATERIALS ENGINEERING

SAN JOSÉ STATE UNIVERSITY

December 2016

Melanie McNeil, Ph.D.

Richard Chung, Ph.D.

Ankit Vora, Ph.D.
Department of Biomedical, Chemical, and Materials Engineering

Department of Biomedical, Chemical, and Materials Engineering

Research Staff Member, International Business Machine Research - Almaden 


\title{
ABSTRACT \\ DIRECTED SELF-ASSEMBLY OF HIGH- $\chi$ BLOCK COPOLYMERS FOR ADVANCED PATTERNING APPLICATIONS
}

\author{
by Gabriela Alva
}

High- $\chi$ block copolymers (BCP) have gained interest to be used as an alternative to currently used multiple patterning techniques for obtaining sub-lithographic features due to their ability to self-assemble at the nanoscale. However, there is a challenge in controlling the orientation of high- $\chi$ BCPs at the air interface. This work describes the use of a formulation-based approach wherein different surface active polymers (SAP) were added as additives to control the orientation of poly(styrene-b-methyl carbonate) (PS-b-PMeCAR) lamellae at the air interface. The resulting thin films made from these formulations showed successful formation of perpendicular lamellae on neutral underlayer substrates upon thermal annealing. The higher surface active SAP demonstrated better orientation control with lower loadings and on thicker films. These films were characterized by atomic force microscopy, grazing incidence small angle xray spectroscopy, and x-ray photoelectron spectroscopy to confirm the perpendicular orientation of the lamellar domains and the distribution of the SAP in the BCP thin film. The vertically oriented BCP domains were used as an etch mask by selectively removing the more etch labile PMeCAR block by reactive ion etching using oxygen plasma. A technique called sequential infiltration synthesis (SIS), followed by removing the PS block to obtain $\sim 9.5 \mathrm{~nm}$ half pitch domains, was also used. Directed self-assembly via graphoepitaxy was also successfully demonstrated. Future work includes investigation of different BCP platforms and morphologies other than lamellae for patterning work. 


\section{ACKNOWLEDGMENTS}

I would like to thank Dr. Melanie McNeil for her help and support throughout my time at San Jose State University. I would also like to thank Dr. Richard Chung for being a part of my committee. I want to thank Dr. Ankit Vora for his help, advice, and support during my time at IBM, and for being a great mentor. I would also like to thank Dr. Kristin Schmidt for her help throughout the project and being another mentor to me at IBM.

Thank you to all the researchers at IBM that were a part of this project and shared their knowledge: Noel Arellano for his help in all the etching processes, Lisa Lofano for SEM imaging, Leslie Thompson for XPS analysis, and Teddie Magbitang for his initial training on sample preparation and SIS process. I would also like to thank Dr. Jane Frommer, Dr. Amanda Engler, and Dr. Kumar Virwani for their help and support at IBM.

I want to thank my friends at IBM who helped make this an experience to never forget. Thank you to my family and loved ones for supporting me all the way and encouraging me to do my best. Last but not least, I would also like to thank Dr. Mayra Padilla and Kristi Lozano, who were my first inspirations to pursue a science education, and have supported me throughout my entire journey since I met them as a young girl. 


\section{TABLE OF CONTENTS}

List of Tables......................................................... viii

List of Figures........................................................... ix

List of Equations...................................................... xiii

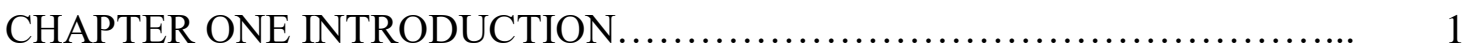

1.1 Background................................................. 1

1.2 Block Copolymers and Directed Self-Assembly.................... 3

1.3 Significance.................................................. 10

CHAPTER TWO LITERATURE REVIEW ................................. 12

2.1 Neutral Substrate Interface......................................... 12

2.2 Air Interface Control......................................... 15

2.2.1 Solvent Vapor Annealing (SVA) ........................ 15

2.2.2 Top-Coat............................................. 17

2.2.3 Selective Chemical Modification............................ 19

2.2.4 Embedded Neutral Layer................................ $\quad 20$

2.3 Etch Processes................................................ 22

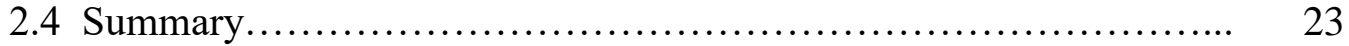

CHAPTER THREE OBJECTIVE AND HYPOTHESIS........................ 25

3.1 Objective and Justification..................................... 25

3.2 Motivation .................................................... 26

CHAPTER FOUR MATERIALS AND METHODS............................. 28

4.1 Materials....................................................... 30

4.2 Methods....................................................... 31

4.2.1 Preparation of Underlayer Solution....................... 31

4.2.2 Preparation of BCP solution and SAP formulations.......... 31

4.2.3 Identification of Neutral Underlayer....................... 31

4.2.4 Thin Film Sample Preparation............................ 32

4.2.5 Preparation of Graphoepitaxy Pre-pattern................. 32

4.2.6 Preparation of Graphoepitaxy DSA Samples............... 33

4.2.7 SIS Process ........................................... 34

4.2.8 Etch Process........................................ 34

4.3 Instrumentation................................................ 35

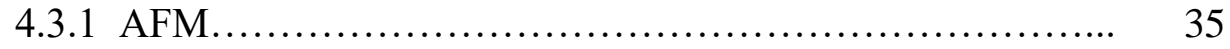

4.3.2 SEM................................................. 35

4.3.3 GISAXS ............................................ 35

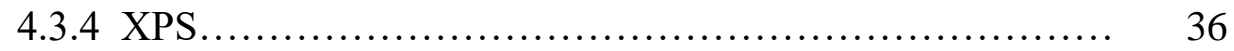


CHAPTER FIVE RESULTS AND DISCUSSION ......................... 37

5.1 Random Copolymer as Neutral Underlayer...................... 37

5.2 Air Interface Control........................................ 39

5.3 Etch Process.............................................. 50

5.4 Directed Self-Assembly using Graphoepitaxy................... 53

CHAPTER SIX CONCLUSIONS...................................... 58

REFERENCES........................................................ 60 


\section{LIST OF TABLES}

Table 1. Table of experiments.................................. 28

Table 2. Design of experiment for time and temperature effect on DSA

alignment................................................. 56 


\section{LIST OF FIGURES}

Figure 1. Graph showing Moore's trend over the last few decades, with the vertical axis representing the number of transistors on a chip at a logarithmic scale and the horizontal axis the year of introduction of each new generation of microprocessors [1] (Reprinted with permission from Elsevier)

Figure 2. A schematic showing the general steps in photolithography [4] (Reprinted with permission from Elsevier).

Figure 3. a) The general structure of a block copolymer with a block of polymer A covalently bonded to a block of polymer B. b) An example of a block copolymer, poly(styrene-b-methyl methacrylate) (PS-b-PMMA).

Figure 4. Phase diagram showing the different morphologies that can be obtained through self-assembly of a linear diblock copolymer [11] (reprinted with permission from the Royal Society of Chemistry)

Figure 5. Diagrams of parallel lamellar orientation due to preferential interactions at the interfaces (a) symmetric and (b) asymmetric, and (c) perpendicular orientation with neutral interfaces, where $n$ is the number of layers, $\mathrm{L}$ and $\mathrm{L}_{0}$ are the lamellar period, and $\mathrm{D}$ is the plate separation [12] (Reprinted with permission from the American Chemical Society. Copyright 1994 American Chemical Society)

Figure 6. Figure 6. Scanning electron microscope images of a prepattern, self-assembled BCP, and aligned BCP when top-down and bottom-up methods are combined [16] (Figure from Ankit Vora. Reproduced with permission)

Figure 7. a) Chemoepitaxy and b) Graphoepitaxy depiction for lamellae morphology DSA [16] (Figure from Ankit Vora. Reproduced

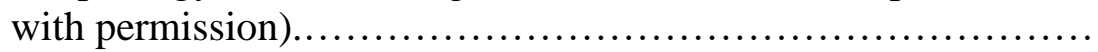

Figure 8. Atomic force microscopy images of two different molecular weight PS-b-PMMA showing that a pitch lower than $20 \mathrm{~nm}$ is not possible [16] (Figure from Ankit Vora. Reproduced with

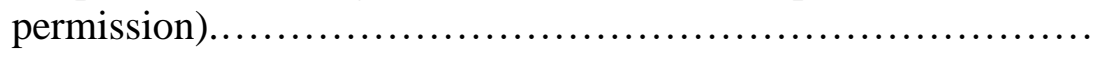


Figure 9. Atomic force microscopy height and phase images (a, b) of PSb-PI on a PS preferential brush. (c) $1 \mathrm{~L}_{0}$ step height expected from both interfaces having preferential wetting. Atomic force microscope height and phase images (d, e) of PS-b-PEI78 on a PS preferential brush. (f) $0.5 \mathrm{~L}_{0}$ step height indicating neutral air interface [27] (reprinted with permission from American Chemical Society. Copyright 2013 American Chemical Society)

Figure 10. Schematic of $0.5 \mathrm{~L}_{0}$ step height formation when the thin film has (a) neutral free surface and preferential substrate or (b) preferential free surface and neutral substrate [27] (reprinted with permission from American Chemical Society. Copyright 2013 American Chemical Society)...........................

Figure 11. Atomic force microscopy images of PdS-b-PI-b-PdS cylinders as solvent removal rate decreases and causes change in domain orientation from parallel to perpendicular (Reprinted with permission from the American Chemical Society. Copyright 2012 American Chemical Society) [34].......................

Figure 12. Cross-sectional SEM images of PS-b-P2VP with three different top coats of PS, PS-r-P2VP, and P2VP respectively (Reprinted with permission from the American Chemical Society. Copyright 2014 American Chemical Society) [6].

Figure 13. Schematic of polarity switching top-coat application process. Substrate surface is modified to be neutral, the BCP, followed by the top-coat, are spin coated on. The BCP and top-coat are then thermally annealed, at which point the top-coat switches polarity. The top-coat is then stripped off to expose perpendicular lamellae [13] (reprinted with permission from The American Association for the Advancement of Science)..........

Figure 14. Scanning electron microscope images of PS-PI as the degree of epoxidation increases on the PI block, showing PS-PI can be converted to a $\mathrm{BCP}$ with equal surface energies of the blocks [15] (reprinted with permission from the American Chemical Society. Copyright 2012 American Chemical Society)..........

Figure 15. X-ray photoelectron spectroscopy and SEM data showing effect of ENL on perpendicular orientation, along with representative cartoons. (a) No ENL shows island/hole morphology, (b) $10 \mathrm{wt}$ $\%$ ENL shows partial perpendicular lamellae coverage, and (c) 20 wt \% ENL shows full coverage [29] (Reprinted with 
permission from the American Chemical Society. Copyright 2016 American Chemical Society).

Figure 16. (a) The structure of the BCP, PS-b-PMeCAR. (b) A schematic of the experimental process, starting with identifying the neutral underlayer, varying the SAP composition and concentration to obtain perpendicular lamellae, and directed self-assembly to orient the lamellae in-plane

Figure 17. Atomic force microscope topographic images of (a) asymmetrical wetting showing holes with $1 \mathrm{~L}_{0}$ step height on PMeCAR preferential UL $(0 \% \mathrm{~S})$, (b) $0.5 \mathrm{~L}_{0}$ step height on neutral UL (20\% S), and (c) symmetrical wetting showing islands with $1 \mathrm{~L}_{0}$ step height on PS preferential UL $(32 \% \mathrm{~S})$, with corresponding height graphs of a surface section to measure height differences.

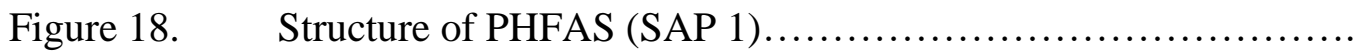

Figure 19. Atomic force microscope phase images of PS-b-PMeCAR + SAP 1 on a modified neutral substrate with (a) $2 \mathrm{wt} \%$ and (b) 3 wt \% SAP 1. Scale bar: $400 \mathrm{~nm}$

Figure 20. Structure of SAP 2, PHFAS-r-PPFS

Figure 21. Atomic force microscope phase images of PS-b-PMeCAR + SAP 2-40 with (a) 2 wt $\%$ and (b) 3 wt \% SAP 2-40: Scale bar: $400 \mathrm{~nm}$.

Figure 22. Atomic force microscope phase images of PS-b-PMeCAR $+2 \%$ SAP 2-40 at film thicknesses (a) $33 \mathrm{~nm}$ and (b) $43 \mathrm{~nm}$. Scale bar: $400 \mathrm{~nm}$.

Figure 23. Atomic force microscope phase images of PS-b-PMeCAR + SAP 2-80 with (a) 2 wt \% SAP 2-80, showing no defects (b) and 3 wt $\%$ SAP 2-80, with flat areas. Scale bar: $400 \mathrm{~nm} . . . \ldots \ldots \ldots . . .$.

Figure 24. Atomic force microscope phase images of PS-b-PMeCAR +2 wt \% SAP 2-80 at different film thicknesses (a) $33 \mathrm{~nm}$ and (b) $43 \mathrm{~nm}$. Scale bar: $400 \mathrm{~nm}$

Figure 25. Plots of GISAXS in-plane scattering profiles for no SAP, SAP 1, SAP 2-40, and SAP 2-80. The sample with no SAP displays a weak 1st order Bragg peak, indicating lack of perpendicular 
lamellae. Samples with SAP display a stronger 1st order Bragg peak, and the presence of 2 nd and 3rd order peaks in a 1:2:3 peak position ratio, typical for lamellae structures

Figure 26. X-ray photoelectron spectra at the fluorine edge showing fluorine content at different detection depths in a control sample with no SAP and a sample with 2 wt $\%$ SAP $2-40 \ldots \ldots \ldots \ldots . . . .$.

Figure 27. X-ray photoelectron spectra at the oxygen edge showing oxygen content at different detection depths in a control sample with no

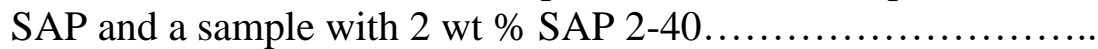

Figure 28. Scanning electron microscope images and corresponding cartoons of PS-b-PMeCAR with 2 wt\% SAP 2-40 (a) before etching, (b) after $\mathrm{O} 2$ etch to remove PMeCar and the underlayer underneath it, (c) after CF4/CHF3 etch to remove SiNx layer, (d) top-down after $\mathrm{O} 2 / \mathrm{N} 2$ etch to remove PS, remaining underlayer, and $\alpha$-carbon layer and, (e) a cross-section.

Figure 29. Scanning electron microscope images and corresponding cartoons of PS-b-PMeCAR +2 wt \% SAP 2-40 a) before SIS process, b) post SIS process, and c) post $\mathrm{O}_{2} \mathrm{RIE}$ etch removing

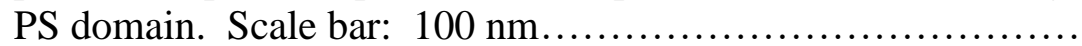

Figure 30. Scanning electron microscope images of (a) the graphoepitaxy pre-pattern and (b) alignment of PS-b-PMeCAR +2 wt $\%$ SAP 2-80 when annealed at $170{ }^{\circ} \mathrm{C}$ for 5 minutes. Scale bar: 100 $\mathrm{nm}$

Figure 31. Scanning electron microscope images of PS-b-PMeCAR $+2 \mathrm{wt}$ $\%$ SAP 2-80 in pre-patterns annealed at (a) $170{ }^{\circ} \mathrm{C}$ for 5 minutes, (b) $200{ }^{\circ} \mathrm{C}$ for 5 minutes, (c) $170{ }^{\circ} \mathrm{C}$ for 10 minutes, and (d) 200 ${ }^{\circ} \mathrm{C}$ for 10 minutes. Scale bar: $100 \mathrm{~nm}$..........................

Figure 32. Design of experiment plots of a) the main effect of time on alignment improvement, and b) the main effect of temperature on alignment improvement..................................

Figure 33. Design of experiment plot for time and temperature interaction on alignment improvement. 


\section{LIST OF EQUATIONS}

Equation 1.

Equation 2. 


\section{CHAPTER ONE}

\section{INTRODUCTION}

\subsection{Background}

"Moore's Law" is an observed trend predicting that the number of transistors on a processor will double per square inch approximately every two years. The trend is shown in Figure 1 [1]. This concept was first introduced in 1965 by Gordon E. Moore, a cofounder of Intel Corporation, after he observed the trend starting with the invention of the integrated circuit (IC) in 1959 [2]. Until now, photolithography is mainly used to produce ICs.

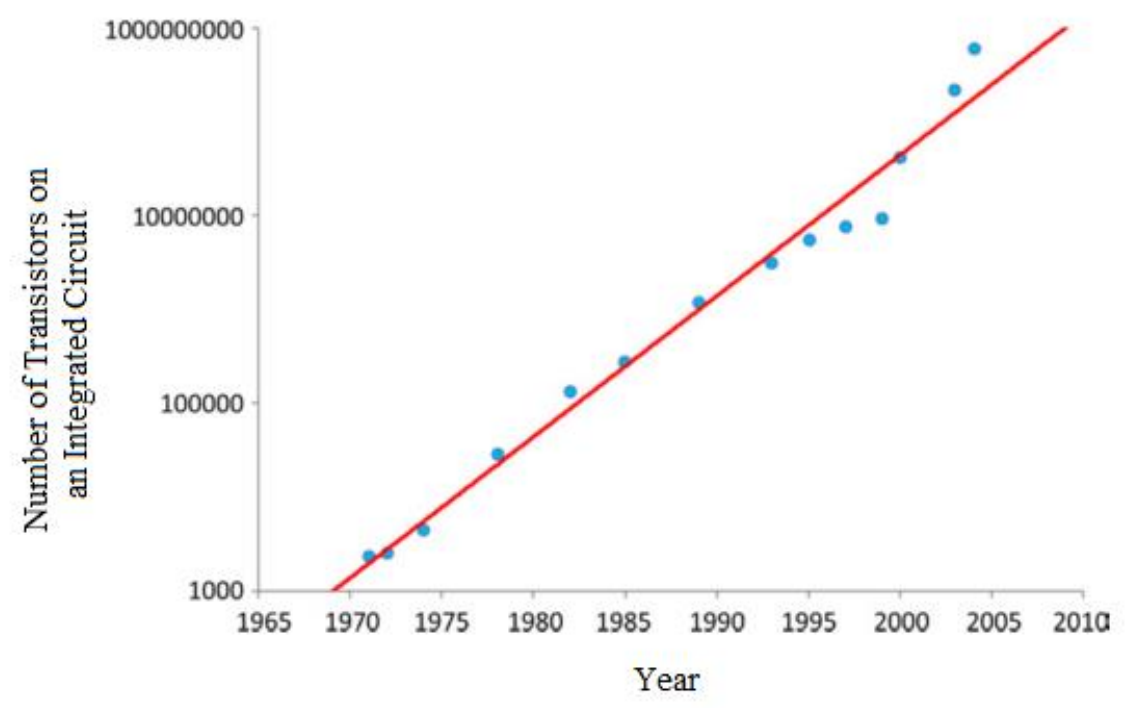

Figure 1. Graph showing Moore's trend over the last few decades, with the vertical axis representing the number of transistors on a chip at a logarithmic scale and the horizontal axis the year of introduction of each new generation of microprocessors [1] (Reprinted with permission from Elsevier). 
Photolithography is a patterning technique using light as a source. The general process is as follows [3]. Light goes through an optical system, and is focused on a mask with the desired pattern on it, which is then projected onto a photosensitive material (photoresist). There are two types of photoresist, positive and negative tone resists. In a positive-tone photoresist, the portions of the resist that are exposed to light become more soluble in the developer (aqueous) and dissolve when developed, while the unexposed portions remain on the substrate. In a negative-tone photoresist, the opposite occurs. The areas exposed to light become insoluble and remain when developed (rinsed with an organic solvent), while the unexposed areas dissolve. The remaining resist acts as an etch mask for pattern transfer into the underlying layers. Figure 2 shows the general process for photolithography [4].

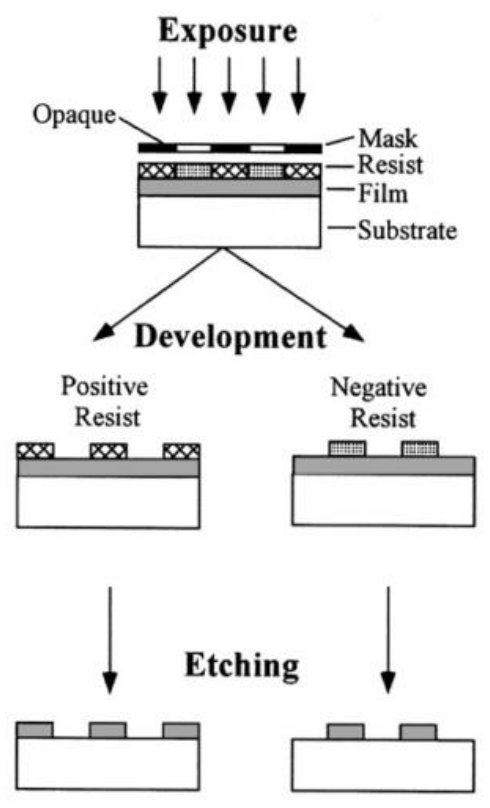

Figure 2. A schematic showing the general steps in photolithography [4] (Reprinted with permission from Elsevier). 
The size of the pattern features is limited by the wavelength of the light source. The smaller the wavelength, the smaller feature size can be reached. Over the years, the industry has progressed from using $436 \mathrm{~nm}$ wavelength lithography to the current $193 \mathrm{~nm}$ wavelength immersion lithography (193i), which replaces the air gap between the substrate surface and final lens with water to increase the refractive index, and therefore resolution. The $193 \mathrm{i}$ wavelength has been used in industry for the past 11 years, with current pitch limit of $65 \mathrm{~nm}$, extending its use through methods such as multiple patterning [5]. Multiple patterning, as the name implies, requires multiple lithographic steps, making the process costly. However, error in the overlay of the mask becomes an issue and critical dimension control becomes more difficult. Continuing the trend of Moore's law of cheaper and smaller ICs is becoming more and more challenging with current lithography technology.

The wavelength of $13.5 \mathrm{~nm}$ available at extreme ultraviolet light (EUV), is one of the next generation methods in line to continue the trend of Moore's law. However, the tool is not yet production ready, and so is unavailable for high volume manufacturing [45]. As such, various alternatives are being investigated to extend current $193 \mathrm{inm}$ photolithography. One promising candidate is the use of block copolymers (BCPs) for directed self-assembly (DSA) as a complimentary method with current top-down photolithography, which was the focus of this study.

\subsection{Block Copolymers and Directed Self-Assembly}

A copolymer is a polymer that is made of two or more chemically different polymer chains (noted as A, B, etc.). In a block copolymer, each monomer forms a 
homopolymer, for example, polymer A and polymer B, which are covalently bonded together. A block copolymer can form various architectures, such as linear diblock or triblock, star, brush or comb-polymers, or even more complex structures [7]. An example of a linear AB diblock copolymer is shown in Figure 3.

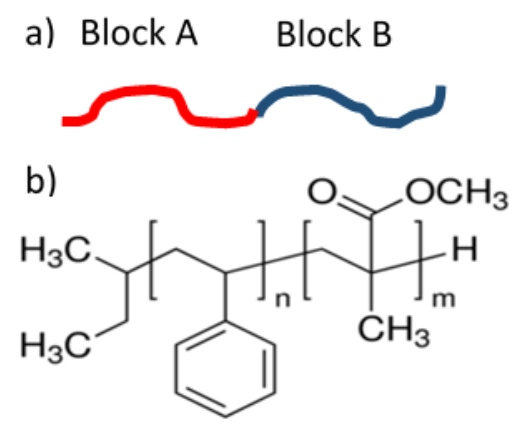

Figure 3. a) The general structure of a block copolymer with a block of polymer A covalently bonded to a block of polymer B. b) An example of a block copolymer, poly(styrene-b-methyl methacrylate) (PS-b-PMMA).

The reason for the interest in BCPs is their ability to self-assemble, which is the spontaneous organization of materials into features (domain periodicity, $\mathrm{L}_{0}$ ) as small as a few nanometers [8]. Because the constituents of BCPs are generally immiscible, the two blocks phase-separate. However, since they are covalently bonded, they can separate only on the microscale. The phase separation is governed by the competing enthalpic (domain interaction at the interface) and entropic (stretching energy of the $\mathrm{BCP}$ chains) interactions of the two BCP components [9-10]. As the enthalpic interaction increases (tries to minimize the domain interaction), phase-separation begins and the BCP selfassembles. The enthalpic interaction is described by the Flory-Huggins interaction parameter, chi $(\chi)$ multiplied by the degree of polymerization $(\mathrm{N})$, and so a higher $\chi \mathrm{N}$ BCP typically has a stronger segregation. 
The BCPs can assemble into various morphologies, such as lamellae, cylinders, and bicontinuous gyroids depending on the relative volume fraction of each block (f), the degree of polymerization $(\mathrm{N})$, and the interaction parameter, $\chi$. A phase diagram showing the dependence of different morphologies on these factors is shown in Figure 4 [11]. In order for microphase separation to occur, the condition, $\chi \mathrm{N} \geq 10.5$, must be met; otherwise, the BCPs will be in a disorganized state (entropic interaction dominates) [9].

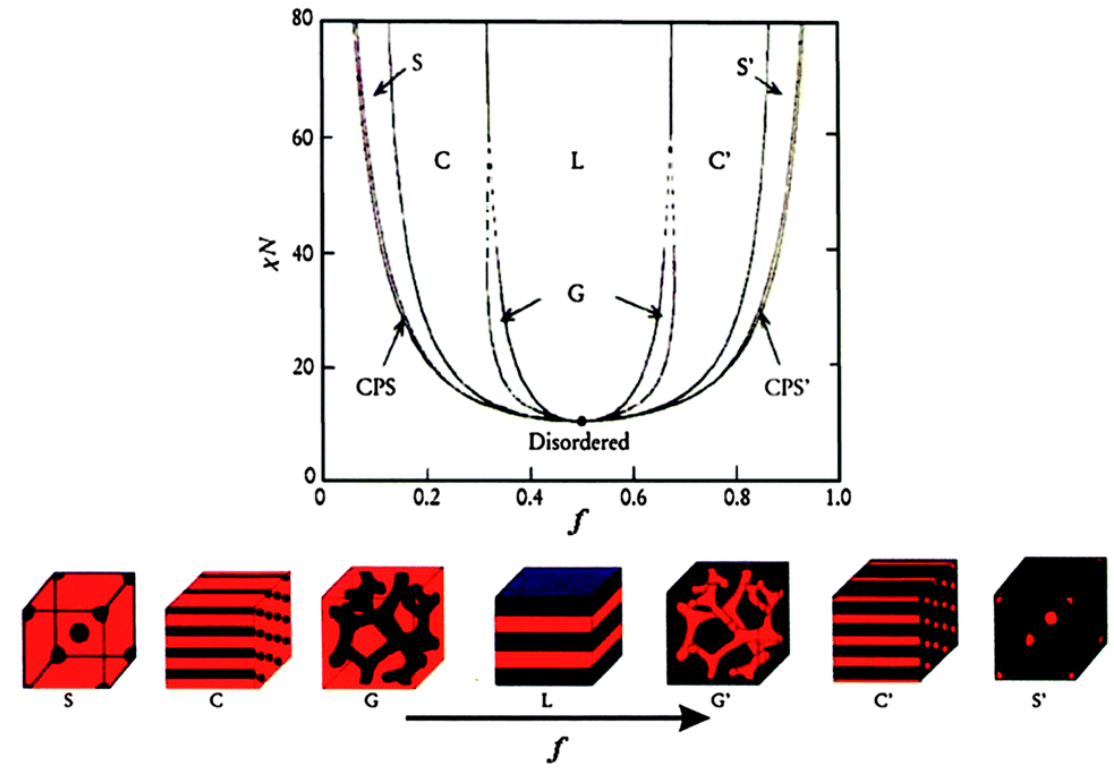

Figure 4. Phase diagram showing the different morphologies that can be obtained through self-assembly of a linear diblock copolymer [11] (reprinted with permission from the Royal Society of Chemistry).

To use lamellae morphology for patterning applications, perpendicular orientation of the lamellae with respect to the substrate is desired to create line space patterns. In a thin film, the orientation of a $\mathrm{BCP}$ is influenced by the interfacial energy between the BCP film and the substrate and air interfaces [9-10]. The interfacial (surface) energy wants to be at a minimum. Because the surface energy is generally different between 
each block and interface, this leads to preferential interaction (wetting) between selective blocks and interfaces to minimize surface energy. For lamellar morphology, the preferential interaction causes orientation parallel to the surface of the substrate [12]. To obtain a perpendicular orientation, both blocks need to have a similar surface energy at the interface, i.e., a neutral interface is required. Figure 5 shows how different wetting conditions influence lamellae orientation, i.e., symmetric wetting (same block is preferential at the substrate and air interface) or asymmetric wetting (different blocks are preferential at substrate and air interface) lead to parallel orientation, while neutral wetting (substrate and air interface are neutral) results in perpendicular orientation [12].

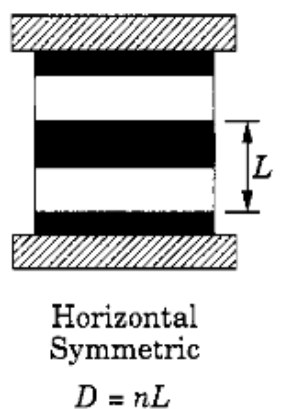

(a)

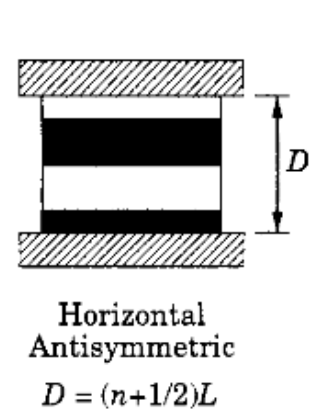

(b)

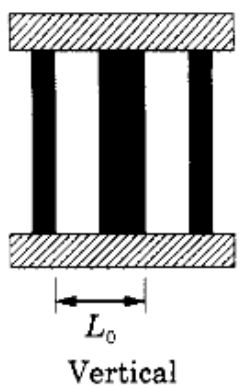

(c)

Figure 5. Diagrams of parallel lamellar orientation due to preferential interactions at the interfaces (a) symmetric and (b) asymmetric, and (c) perpendicular orientation with neutral interfaces, where $\mathrm{n}$ is the number of layers, $\mathrm{L}$ and $\mathrm{L}_{0}$ are the lamellar period, and $\mathrm{D}$ is the plate separation [12] (Reprinted with permission from the American Chemical Society. Copyright 1994 American Chemical Society).

A neutral substrate interface is typically achieved using a neutral underlayer, which uses random copolymers with similar surface energies as the BCP blocks, which are composed of similar constituents of the $\mathrm{BCP}$. After modifying the substrate to be neutral, the BCP can be spin coated onto the modified substrate to create a thin film [13- 
15]. The film is then thermally annealed above the $T_{g}$ (i.e., glass transition) temperature, but below the decomposition temperature, providing the mobility for the $\mathrm{BCP}$ to reorganize itself into the predicted ordered state [10].

Neutral underlayers are a way to control BCP orientation in the out-of-plane direction. For BCPs to be useful as an alternative patterning technique, in-plane alignment is needed as well. By combining current top-down lithography technology, which has good long range order but relatively larger features, and bottom-up selfassembly, which has short range order, but small features, the self-assembly can be directed, as demonstrated in Figure 6 [16]. There are currently two main forms for directing the self-assembly of BCPs: chemoepitaxy and graphoepitaxy [17].
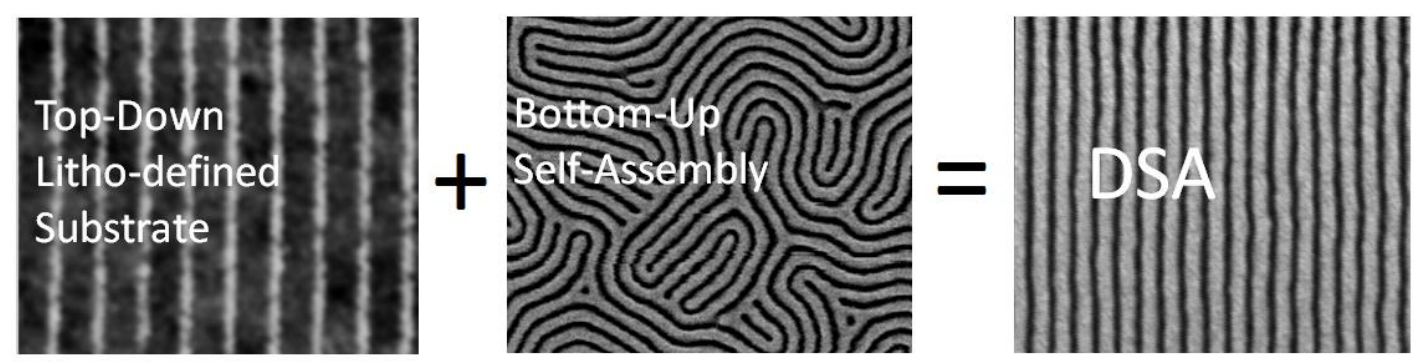

Figure 6. Scanning electron microscope images of a pre-pattern, self-assembled BCP, and aligned BCP when top-down and bottom-up methods are combined [16] (Figure from Ankit Vora. Reproduced with permission).

Chemoepitaxy incorporates a top-down process (e.g., e-beam, 193i, etc.) to create a pre-patterned substrate for the bottom-up assembly of the BCP to follow. It uses a chemical guiding pattern to direct the self-assembly of the BCP, with pinning stripes made of a material that can preferentially attract one of the BCP components. The stripes are interspaced by an integer multiple of the pitch of the BCP. In between the pinning 
stripes is a neutral material that equally attracts both components. The BCP can align along the pinning stripe, and the rest of the BCP alignment will follow [17-18]. The pinning stripes are preferred to be as close to $0.5 \mathrm{~L}_{0}$ or $1.5 \mathrm{~L}_{0}$ in width to minimize the interaction between the pining stripe and the component of the block copolymer that is not attracted to it. This process is depicted in Figure 7a [16].

Graphoepitaxy uses a topographic pre-pattern to guide alignment of the BCP. It uses lithography (e.g., e-beam, 193i, EUV, etc.) to create the topographic pre-pattern (e.g. trenches, holes, etc.) from photoresist materials. The photoresist will typically have a preferential interaction with one of the components of the $\mathrm{BCP}$, to help guide alignment in-plane, along the pre-pattern features. For trenches, inside the topographic pre-pattern, the bottom is neutral so that the BCP orients perpendicular to the substrate for out-ofplane alignment [18-19]. The process for this method using trenches for the pre-pattern is shown in Figure $7 b$ [16].

a) Chemoepitaxy
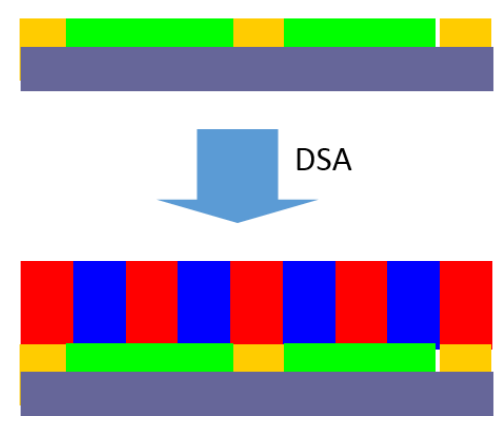

b) Graphoepitaxy

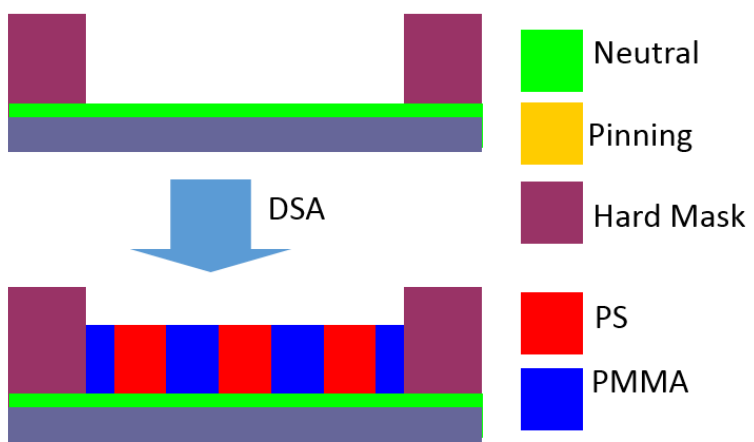

Figure 7. a) Chemoepitaxy and b) Graphoepitaxy depiction for lamellae morphology DSA [16] (Figure from Ankit Vora. Reproduced with permission). 
Once the orientation of the BCP is controlled in and out-of-plane, the thin film can be used as a patterning template, similar to the role of a photoresist in lithography [10]. After the BCP morphology and orientation is controlled into a desired pattern template, one of the block components is removed, typically through an etch process, to create a mask for pattern transfer.

Currently, PS-b-PMMA is dominantly used for DSA applications in industry. The structure is shown in Figure 3b. A tremendous amount of research has been done on PS-b-PMMA for DSA and it is currently the most understood system. It is a readily available material and has shown to have simple processing conditions ideal for fabrication processes as it can be spin-coated from fab-friendly solvents (e.g., PGMEA) and can be thermally annealed [21-22]. Additionally, the surface energies of the two blocks are balanced at elevated temperatures at the free surface, enabling perpendicular lamella to form when on a neutral substrate surface. Tsai et al. demonstrated the first 29 nm pitch DSA patterned FinFET device using a process which can easily be integrated into standard device fabrication [21].

However, PS-b-PMMA is limited by a relatively low $\chi$ and therefore; a resolution limit of $20 \mathrm{~nm}$ full pitch $\left(\mathrm{L}_{0}\right)$. Figure 8 demonstrates the pitch limit of PS-b-PMMA using AFM images of two different molecular weight PS-b-PMMA BCPs, one with a 20 $\mathrm{nm}$ pitch and molecular weight of $30 \mathrm{~K}$, and another with a lower molecular weight of 28 $\mathrm{K}$ [16]. In order for DSA to be competitive with current photolithography standards, it is necessary to reach a higher resolution (i.e., smaller pitch). This can be achieved by 
controlling the degree of polymerization $(\mathrm{N})$ and the $\chi$ parameter. Equation 1 shows the relationship between $\chi, \mathrm{N}$, and $\mathrm{L}_{0}$, where $\alpha$ is a statistical segment length [6].

$$
\mathrm{L}_{0} \approx \alpha \mathrm{N}^{2 / 3} \chi^{1 / 6} \quad \text { Equation } 1
$$

By increasing the $\chi$ parameter and reducing $\mathrm{N}$, a smaller pitch is possible, making high- $\chi$ BCPs of interest. However, although PS-b-PMMA is inherently neutral at the air interface, high- $\chi$ BCPs are usually not, and so the challenge of using high- $\chi$ BCPs is the difficulty in orientation control at the air interface.
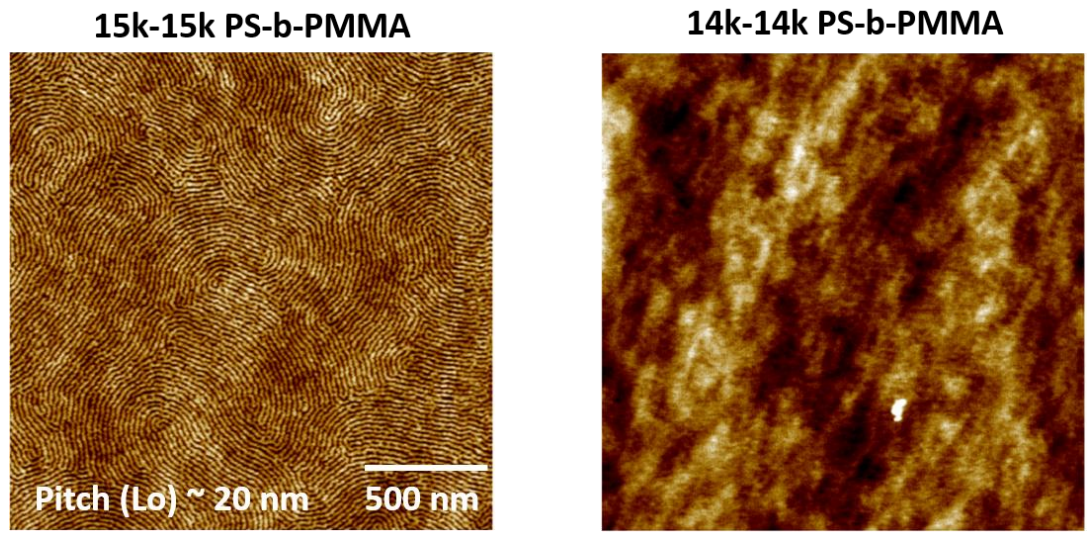

Figure 8. Atomic force microscopy images of two different molecular weight PS-bPMMA showing that a pitch lower than $20 \mathrm{~nm}$ is not possible [16] (Figure from Ankit Vora. Reproduced with permission).

\subsection{Significance}

Lithography is a costly process, especially with the current use of multiple patterning, requiring multiple lithographic steps to reach sub-20nm features. The ability of BCPs to self-assemble at the nanoscale make them of interest to use as masks with current photolithography processes. High- $\chi$ BCPs are of particular interest to surpass the $20 \mathrm{~nm}$ pitch limit of PS-b-PMMA, which is the dominantly used BCP for DSA in 
industry. If an orientation control method compatible with fabrication friendly conditions can be achieved, then high- $\chi$ BCPs can be exploited to form nanoscopic features smaller than those offered by current photolithography processes at a lower cost. 


\section{CHAPTER TWO}

\section{LITERATURE REVIEW}

The idea of using block copolymer self-assembly for nanolithographic applications was first introduced by Mansky in 1995 in a publication, "Monolayer films of diblock copolymer microdomains for nanolithographic applications [22]." Since then, a variety of research has been done on self-assembly and DSA. For DSA to be successful, control of domain orientation of the BCP thin films is crucial. For perpendicular orientation, it is necessary for the interfaces to be neutral. In this chapter, a literature overview for methods of orientation control of BCPs and etching methods is given.

\subsection{Neutral Substrate Interface}

One of the earlier studies on using random copolymers to modify the substrate interface was done by Kellogg et al., in which random copolymers consisting of PS and PMMA (PS-r-PMMA) were coated onto substrate interfaces [23]. This study showed that as the specific interaction between either block of the BCP and the substrate decreased, perpendicular orientation was achieved at the substrate interface.

Later on, in 1997, Mansky et al. published a study on using random copolymers for substrate modification, but instead used hydroxyl $(\mathrm{OH})$ end grafted PS-r-PMMA and identified surface neutrality through contact angle measurements [24]. The use of the $\mathrm{OH}$ end group on the random copolymer allows the copolymer to end-graft itself to the silicon oxide layer of the substrate, and therefore immobilizes it. The reason for using end-grafted polymers was because previously the random copolymer chains were not 
chemically linked to the substrate, and so the copolymer could diffuse into the BCP film during annealing.

Mansky et al. showed that when a constant incommensurate film thickness is used, $1 \mathrm{~L}_{0}$ ("classical") step height features that look like islands or holes are seen at the surface [25]. Depending on film thickness and whether there is symmetric or asymmetric wetting, either island or hole topography will be seen. Peter et al. took this a step further to identify the neutrality of self-assembled monolayers (SAMs) [26]. The SAM surfaces were oxidized through $\mathrm{x}$-ray radiation with a range of exposures. When a transition from islands to holes was seen, it was interpreted as a switch of the block that preferentially wet the substrate.

In 2013, Kim et al. reported a new phenomenon in which $0.5 \mathrm{~L}_{0}$ step height islands and holes were observed [27]. In the study, two different lamella-forming high- $\chi$ BCPs were used, poly(styrene-block-[isoprene-random-epoxysoprene]) (PS-b-PEI78) and poly(4-trimethylsilelstyrene-block-D,L-lactide) (PTMSS-b-PLA). The BCP, PS-bPEI78, is PS-b-PI with 78 mol \% epoxidation on the isoprene block which makes the BCP neutral at the air interface. When PS-b-PI and PS-b-PEI78 were coated onto a PS brush, which is preferential to the PS block, PS-b-PI formed classical $1 \mathrm{~L}_{0}$ step height features, while PS-b-PEI78 formed 0.5L $\mathrm{L}_{0}$ step height features, shown in Figure 9 [27]. 


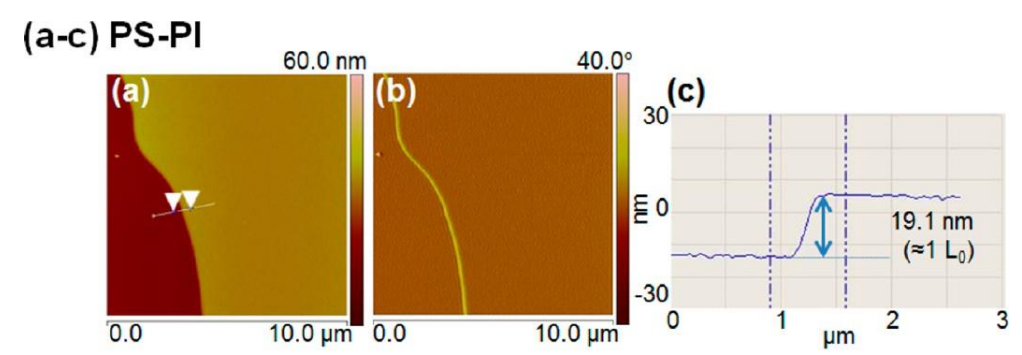

(d-f) PS-PEI78

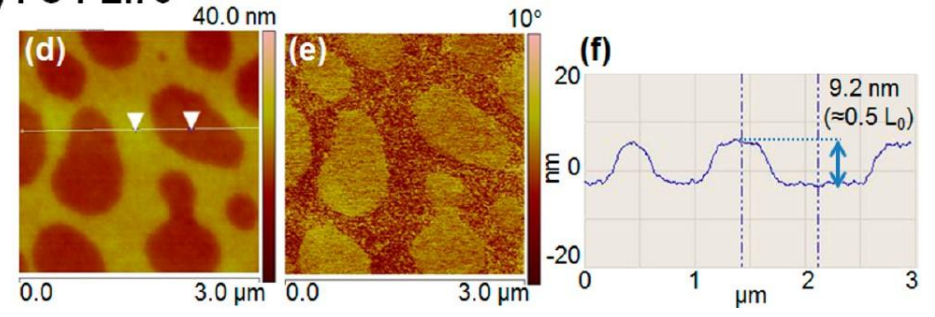

Figure 9. Atomic force microscopy height and phase images (a, b) of PS-b-PI on a PS preferential brush. (c) $1 \mathrm{~L}_{0}$ step height expected from both interfaces having preferential wetting. Atomic force microscope height and phase images (d, e) of PS-b-PEI78 on a PS preferential brush. (f) $0.5 \mathrm{~L}_{0}$ step height indicating neutral air interface [27] (reprinted with permission from American Chemical Society. Copyright 2013 American Chemical Society).

The inverse experiment was done using PTMSS-b-PLA, where PTMSS

preferentially wets the air interface. When it was annealed on a neutral substrate, $0.5 \mathrm{~L}_{0}$ topography was also observed. Figure 10 shows a schematic that illustrates the $0.5 \mathrm{~L}_{0}$ step heights that result from thin films that have only one neutral interface [27].

(a) Neutral Free Surface Preferential Substrate

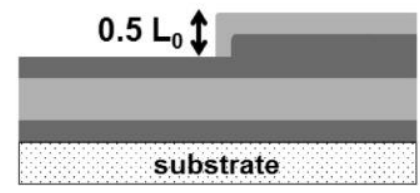

(b) Preferential Free Surface Neutral Substrate

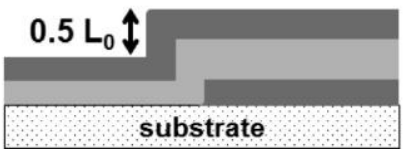

Figure 10. Schematic of $0.5 \mathrm{~L}_{0}$ step height formation when the thin film has (a) neutral free surface and preferential substrate or (b) preferential free surface and neutral substrate [27] (reprinted with permission from American Chemical Society. Copyright 2013 American Chemical Society.). 


\subsection{Air Interface Control}

The challenge of using high- $\chi$ BCPs is that due to the large difference in the interaction parameter between the blocks, it is likely that one block will be preferential at the air interface in order to minimize surface energy [28]. Various high- $\chi$ BCPs have been proposed and studied to use for DSA, including: poly(styrene-block 2-vinyl pyridine) (PS-b-P2VP) [14][29], poly(styrene-block dimethylsiloxane) (PS-b-PDMS) [30], poly(styrene-block-ethylene oxide) (PS-b-PEO) [31], and poly(styrene-blocktrimethylene carbonate) (PS-b-PTMC) [32]. To avoid the formation of a parallel layer at the air interface for these high- $\chi$ BCPs, different orientation control methods have been developed, including solvent vapor annealing (SVA), top-coat, and chemical modification of BCPs.

\subsubsection{Solvent Vapor Annealing (SVA)}

When a BCP thin film is cast onto a substrate, it usually becomes kinetically trapped in a non-equilibrium, and often, disorganized state. In order for BCPs to selfassemble into their equilibrium structure, the $\mathrm{BCP}$ chains must be mobile enough to reorganize themselves. Typically, simple thermal annealing above $\mathrm{T}_{\mathrm{g}}$ can facilitate the attainment of the organized structures at equilibrium. However, long anneal times are often required, and because of the surface energy difference of the blocks, a layer of the material with the lowest surface energy forms at the free surface [33]. In response to this, various groups have employed solvent vapor annealing (SVA) for orientation control [31], [34]. In SVA, a mobile polymer film is formed by exposing the as-prepared BCP thin film to one or more solvent vapors in a solvent exposure chamber at temperatures 
typically below the $T_{g}$ of either block [35]. The solvent vapors cause the thin film to swell and become mobile. Removal of the solvent vapors kinetically traps the polymer, preserving the orientation. One of the first examples using SVA was completed by Albalak et al. in 1998 [36]. Since then, SVA has been successfully used for orientation control of high- $\chi$ BCPs, such as PS-b-PDMS [30], PS-b-P2VP [37], and PS-b-PEO [31].

Albert et al. studied the effect of solvent evaporation rate on poly(deuterated styrene-b-isoprene-b-deuterated styrene) (PdS-b-PI-b-PdS) triblock copolymer [34]. Using a constant concentration of chloroform as the solvent vapor, parallel cylinders formed. However, depending on the solvent removal rate, orientation changed. At instantaneous removal, morphology and orientation were conserved, but as the rate decreased, the orientation at the free surface switched to perpendicular, with featureless regions observed at intermediate removal rates. Figure 11 shows AFM images of the free surface as solvent removal rate decreased [34].

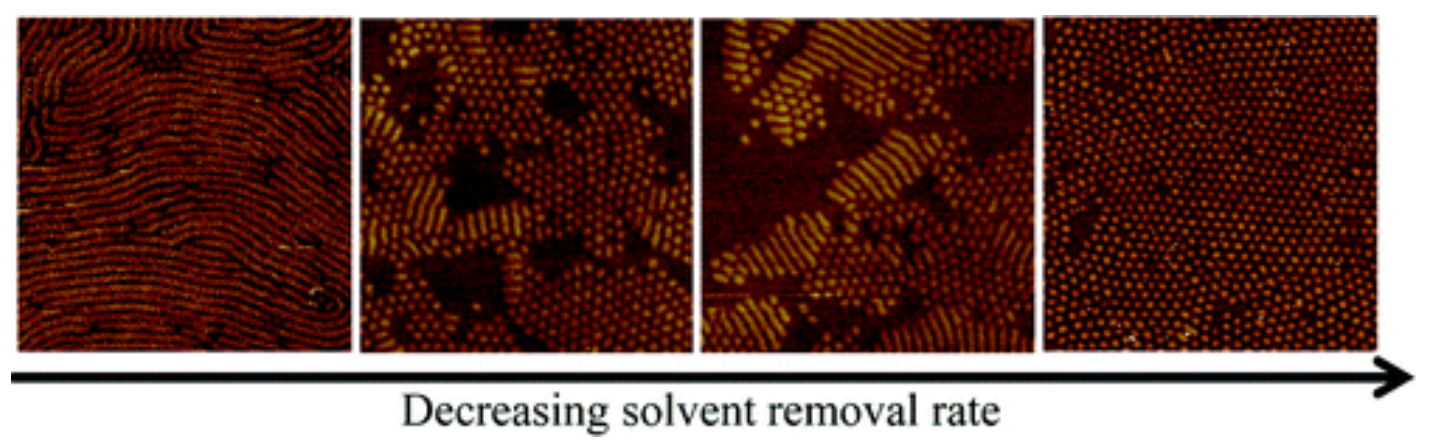

Figure 11. Atomic force microscopy images of PdS-b-PI-b-PdS cylinders as solvent removal rate decreases and causes change in domain orientation from parallel to perpendicular (Reprinted with permission from the American Chemical Society. Copyright 2012 American Chemical Society) [34]. 


\subsubsection{Top-Coat}

A top-coat is compatible with thermal annealing and is meant to work similarly to the neutral substrate underlayer. A material that is non-preferential for both blocks is placed on top of the BCP film at the air interface (e.g., spin coat or polymer off-set printing) so that perpendicular orientation is induced at the air interface with the use of thermal annealing [13-14]. The use of top-coats has been successful on high- $\chi$ BCPs such as PTMSS-b-PLA [13] and PS-b-P2VP [14].

Yoshida et al. reported a study on the use of top-coats for orientation control of PS-b-P2VP lamellae [14][6]. The materials PS, PS-r-P2VP, and P2VP were cross-linked on separate substrates to use as top-coats. The top-coats were then transferred onto the surface of a PS-b-P2VP thin film using either floating or a polymer off-set printing method. Once the top-coat was transferred, thermal annealing was used at $190{ }^{\circ} \mathrm{C}$ for 24 hours. The results showed clear formation of a wetting layer at the top interface when PS and P2VP were used as at the top-coats. When PS-r-P2VP was used as the top-coat, perpendicular lamellae formed. Figure 12 shows cross-sectional SEM images of the three samples [6].

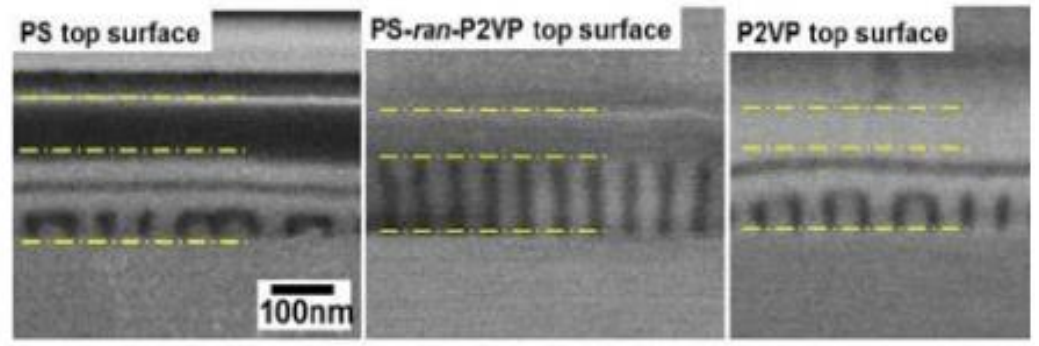

Figure 12. Cross-sectional SEM images of PS-b-P2VP with three different top coats of PS, PS-r-P2VP, and P2VP respectively (Reprinted with permission from the American Chemical Society. Copyright 2014 American Chemical Society) [6]. 
Bates et al. used a different method to obtain perpendicular orientation with the use of a top-coat [13]. In the study, they used a random copolymer containing maleic anhydride, which in its polar form (ring-opened maleic anhydride) is soluble in aqueous ammonium hydroxide. The solution was spin coated onto the surface of the PTMSS-bPLA thin film and was subsequently thermally annealed, during which ring-closure was induced, switching the polarity of the top-coat. This gave a less polar top-coat that is then non-preferential to either block in the $\mathrm{BCP}$, allowing for perpendicular orientation to occur. The top-coat can be stripped after annealing using an aqueous solvent, exposing the BCP for further processing. This process is shown in Figure 13 [13]. The top-coat surface interaction is further tuned through the use of additional co-monomers, like fluorinated methacrylate, to the maleic anhydride. Norbornene was used in order to increase the $T_{g}$ of the top-coat.

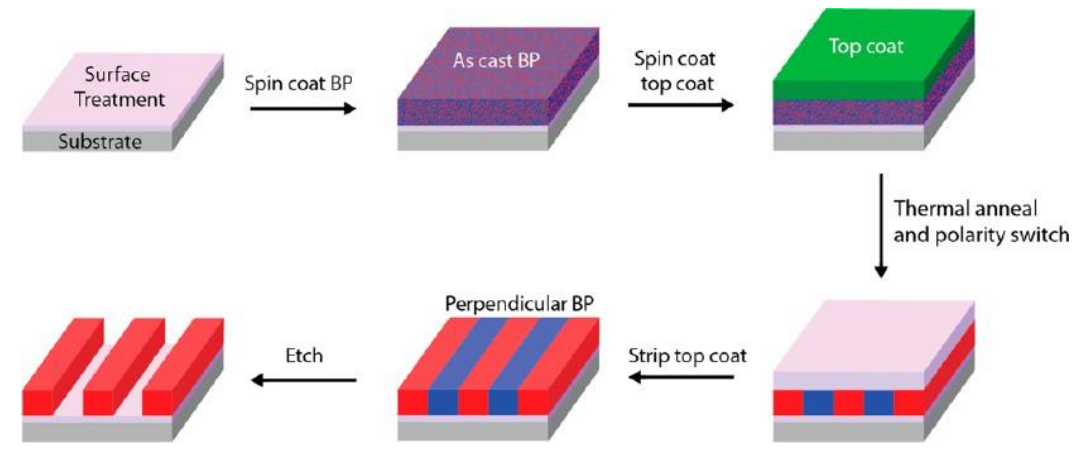

Figure 13. Schematic of polarity switching top-coat application process. Substrate surface is modified to be neutral, the BCP, followed by the top-coat, are spin coated on. The BCP and top-coat are then thermally annealed, at which point the top-coat switches polarity. The top-coat is then stripped off to expose perpendicular lamellae [13] (reprinted with permission from The American Association for the Advancement of Science). 
The floating method for applying a top-coat suffers from scalability issues and introduction of defects [38]. Additionally, neutral polymers spin coated onto the BCP tend to be soluble in a solvent that dissolves the BCP, making removal of the top-coat a challenge. Although Bates et al. surpassed these challenges with the polarity switching top-coat, the process still requires an additional spin coating step and removal step [13].

\subsubsection{Selective Chemical Modification}

Though both SVA and top-coat methods have been shown to induce perpendicular orientation, it would be beneficial to use BCPs that are non-preferential at the air interface and do not require any additional processing steps. Some examples of such BCPs include PS-b-PLA [39], poly(ethylene glycol-b-fluorinated methacrylate) [40], and PS-b-PEI78 [15]. However, design of such BCPs is challenging.

A study on the partial epoxidation of PI in a PS-b-PI BCP was done by Kim et al. [15]. Epoxidation was done post-polymerization of PS-b-PI via a reaction with dimethyl dioxirane. The epoxidation was assumed to occur randomly throughout the PI block. The varied parameter in this study was the degree of epoxidation and its effect on the $\mathrm{BCP}$ interfacial wetting properties and surface energy. Using contact angle measurements, a linear relationship was shown between the degree of epoxidation and surface energy of the PI block. As epoxidation increases, surface energy decreases and reaches a surface energy nearly equal to that of PS, allowing for perpendicular lamellae orientation, shown in Figure 14 [15]. This study showed that the incorporation of a random copolymer into one of the blocks of a $\mathrm{BCP}$ can decouple bulk thermodynamics of the BCP. 

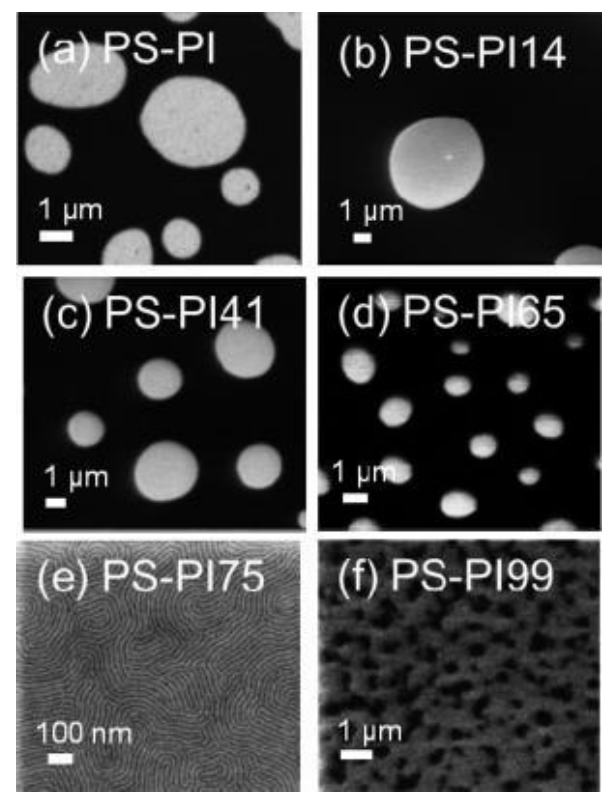

Figure 14. Scanning electron microscope images of PS-PI as the degree of epoxidation increases on the PI block, showing PS-PI can be converted to a BCP with equal surface energies of the blocks [15] (reprinted with permission from the American Chemical Society. Copyright 2012 American Chemical Society).

\subsubsection{Embedded Neutral Layer}

A different approach for orientation control was recently developed by Hustad $e t$ al., through the use of low surface energy polymers [29]. They used a formulation based approach, where they blended a low surface energy BCP into their high- $\chi$ PS-b-P2VP formulation, which they refer to as an embedded neutral layer (ENL). The material used for the ENL was a BCP designed to be both neutral to PS-b-P2VP, and surface active. Surface activity was achieved by using fluorinated polymers, as they are known to have very low surface energies [41]. Since the ENL material is surface active, it segregates to the air-BCP interface during annealing and becomes a neutral layer in order to obtain the desired perpendicular lamellae. Different concentrations of the ENL material (10 and 20 wt \% relative to PS-b-P2VP) were added and evaluated by SEM and XPS, with their 
results shown in Figure 15 [29]. The SEM results revealed that perpendicular lamellae formed with $20 \mathrm{wt} \%$ ENL material, while when $10 \mathrm{wt} \%$ was used, only partial coverage was seen. The XPS results showed that fluorine content was high at the surface of the film. At a higher concentration, a thinner and more defined fluorine containing film was formed, indicating less mixing of the additive in the bulk of the film. This showed that the concentration of the fluorine containing material in the BCP formulation was critical for orientation control.
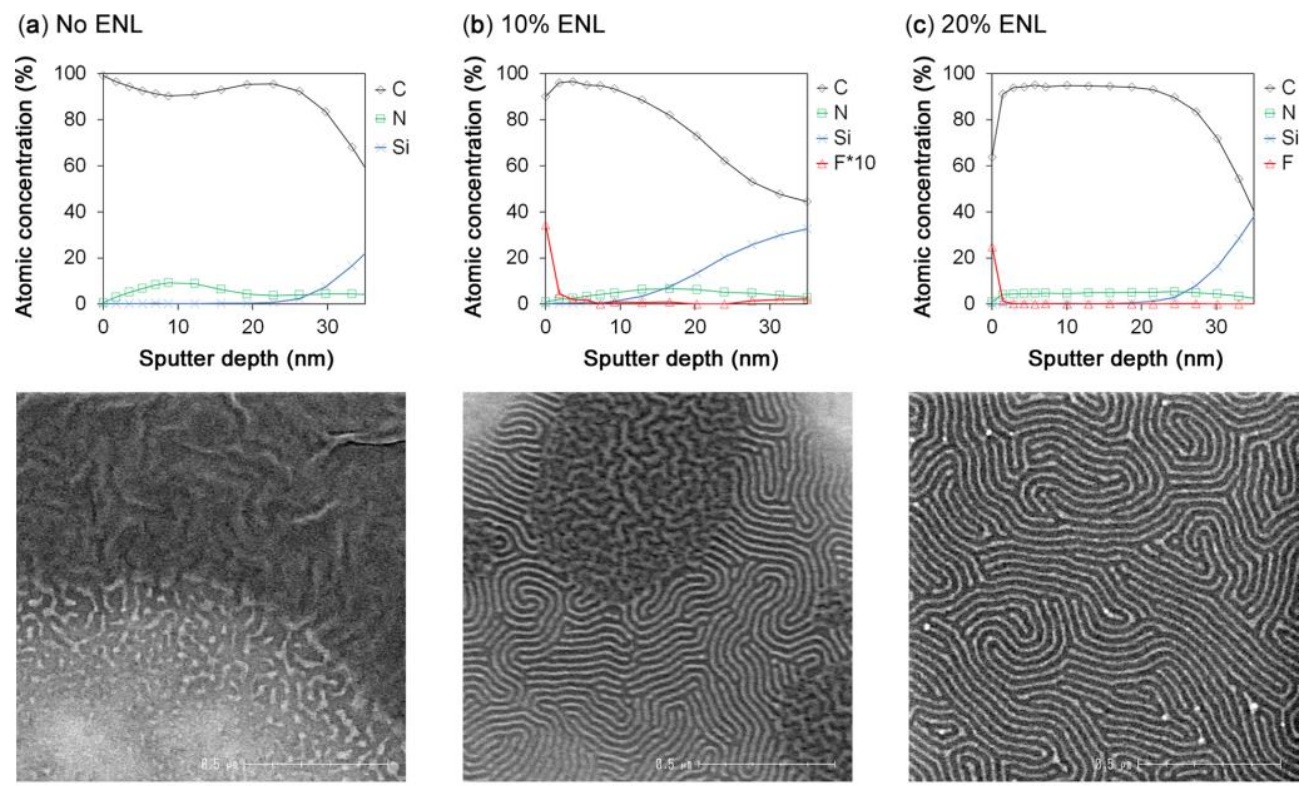

island/hole
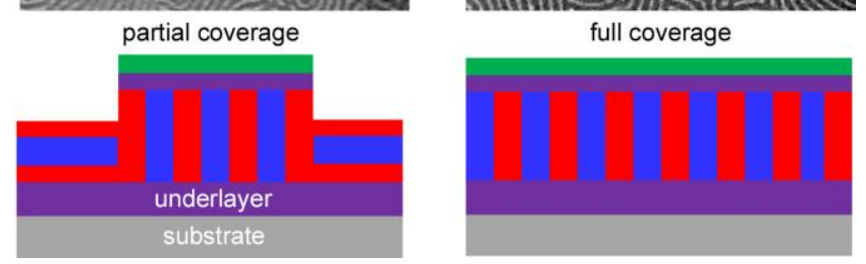

Figure 15. X-ray photoelectron spectroscopy and SEM data showing effect of ENL on perpendicular orientation, along with representative cartoons. (a) No ENL shows island/hole morphology, (b) 10 wt \% ENL shows partial perpendicular lamellae coverage, and (c) $20 \mathrm{wt} \%$ ENL shows full coverage [29] (Reprinted with permission from the American Chemical Society. Copyright 2016 American Chemical Society). 


\subsection{Etch Processes}

For DSA to be successfully implemented as an alternative to current multiple patterning processes, pattern transfer is essential. Reactive ion etching (RIE) is a commonly used process for pattern transfer to selectively remove one of the blocks [3132], [43-44]. Synthesizing BCPs with one etch-resistant block has been one of the strategies focused on by many groups in order to increase etch selectivity for reactive ion etching (RIE) [6]. Typically, the etch resistant block has an inorganic component, such as silicon. A reason Bates et al. selected PTMSS-b-PLA for their top-coat study, was because PTMSS (silicon-containing block) has a much lower $\mathrm{O}_{2}$ RIE rate than PLA [13]. Another high- $\chi$ BCP, PS-b-PDMS, has also been of interest due to the high etch selectivity between the blocks due to the silicon containing block (PDMS) [30].

An alternative approach to increase etch contrast, is the use of sequential infiltration synthesis (SIS). The SIS experiment is carried out in an atomic layer deposition (ALD) chamber, where a material of interest nucleates within the polymer bulk, and these sites then serve as seeds for growth of inorganic particles [44]. Tseng et al. used gas-phase tri-methyl aluminum (TMA) to diffuse through a PS-b-PMMA film and selectively grow in the PMMA domains due to a chemical interaction between TMA and the carbonyl group in PMMA [45]. The process was done via an exposure/purge process in an ALD chamber using TMA and water [44-45]. The presence of the inorganic component, in that case, aluminum oxide, in the PMMA domain made it significantly more etch resistive than PS. 
In a study done by Gokan et al. the dry etch resistance for organic molecules was investigated [46]. Using oxygen-ion etching, they looked at various organic polymers, with particular attention to the carbon and oxygen content in the polymer structure. Carbon was reported to be the rate-determining step in the etch rate, while an increased oxygen content in the polymer structure gave higher etching rates. They found a linear correlation between etch rate and oxygen and carbon content in organic polymers given in Equation 2 [46]

$$
V \propto \frac{N}{N_{C}-N_{O}} \quad \text { Equation } 2
$$

where $\mathrm{V}$ is the etch rate, $\mathrm{N}$ is the total number of atoms in a monomer unit, $\mathrm{N}_{\mathrm{C}}$ is the total number of carbon atoms in a monomer unit, and $\mathrm{N}_{\mathrm{O}}$ is the total number of oxygen atoms in a monomer unit. This means that organic polymers with a high ratio of oxygen to carbon atoms will have a higher etch rate than polymers consisting of mostly carbon.

\subsection{Summary}

Based on previous research, it is now widely known that using end grafted random copolymers consisting of the same or similar constituents as the $\mathrm{BCP}$ can be used to modify the substrate surface to yield a neutral surface. With the work done by Kim et al., the composition of the random copolymer needed to obtain a neutral substrate surface can be easily recognized through the use of AFM by identifying which composition forms $0.5 \mathrm{~L}_{0}$ step height features [27].

Currently, there are three main methods for orientation control of the air-BCP interface: SVA, top-coats, and chemical modification. For SVA, the literature showed that various parameters need to be controlled, and very volatile solvents (e.g., chloroform 
and THF) are typically used in the process [36-37]. Top-coats are compatible with the thermal annealing process, but still require additional processing steps in comparison to PS-b-PMMA [13-14]. More recently, the use of surface active polymers, particularly fluorinated polymers, have become of interest. When using surface active polymers, concentration is an important parameter to consider, as an insufficient amount does not allow for full coverage of perpendicular lamellae on the surface.

To selectively remove one of the blocks of the $\mathrm{BCP}$, the two $\mathrm{BCP}$ constituents must have significantly different RIE rates. Using silicon-containing BCPs has been reported to increase etch selectivity [13], [30]. Use of SIS is another reported method, most commonly done using TMA and water, where the TMA selectively interacts with functional groups, such as carbonyls [43-44]. For organic BCPs, Gokan et al. showed that a high oxygen to carbon ratio is desired [46]. 


\section{CHAPTER THREE}

\section{OBJECTIVE AND HYPOTHESIS}

\subsection{Objective and Justification}

For this study, a carbonate platform BCP, poly(styrene-b-methyl 5-methyl-2-oxo1,3-dioxane-5-carboxylate) (PS-b-PMeCAR), was chosen as a high- $\chi$ BCP for sub-20 nm pitch patterning. Various cyclic carbonate monomers can be polymerized by ringopening polymerization from a hydroxyl-end function PS macroinitiator in order to tune the $\chi$ parameter to PS. Depending on the carbonate monomers used, these carbonate based BCPs can be high- $\chi$ systems. In addition, PMeCAR is expected to have a higher RIE rate due to the high oxygen to carbon ratio of the monomer unit in comparison to PS. Because of the polarity difference between the two block components, they have different surface energies, causing preferential interactions at the substrate and the air interfaces, and therefore an orientation control strategy is needed to enable vertically orientated BCP domains.

The objective of this study was to show how surface active polymers (SAP) as additives can be used to control the orientation of BCPs at the air interface. The SAPs consist of fluorinated groups to achieve a low surface energy, and an alcohol group that allows hydrogen bonding to occur preferentially between the SAP and a functional group in the higher surface energy block, i.e., carbonyl block [40-41]. Once bonded, the SAP is expected to pull this block towards the surface so that both blocks will be at the air interface. 
The goal of this study was to optimize the BCP and SAP system to find fabrication friendly process conditions (e.g., short thermal anneal time, spin coat from conventional solvents, no topcoat, etc.) for enabling perpendicular orientation of the BCP. This was done by analyzing the effect of different SAP compositions and concentrations on the high- $\chi$ BCP orientation using AFM, SEM, and GISAXS. The SAP location in the film was verified using XPS. In addition, film thickness and etch studies with and without sequential infiltration synthesis (SIS) were done. The other objective was to successfully demonstrate DSA using graphoepitaxy. Temperature and annealing time were adjusted to improve alignment.

\subsection{Motivation}

Current methods of orientation control for high- $\chi$ BCPs with sub-20 nm pitch all have their drawbacks in comparison to the more fabrication-friendly method used for PSb-PMMA, such as:

- The solvents used for solvent vapor annealing (SVA) are not fabrication friendly.

- Top-coats require additional processing steps in comparison to PS-b-PMMA.

- Block copolymers inherently neutral at the air interface are challenging to design.

- Embedded neutral layer (ENL) requires a high dose of the surface active neutral polymer, which could change the effective volume fraction of the BCP domains and negatively influence the etch selectivity. 
The use of SAP as additives (i.e., less than $5 \mathrm{wt} \%$ relative to the BCP solids) is a formulation-based method compatible with thermal annealing and without additional processing steps, making the method fabrication-friendly. 


\section{CHAPTER FOUR}

\section{MATERIALS AND METHODS}

In this chapter, the materials, methods, and experimental details used to determine which SAP composition and concentration are ideal for orientation control of the PS-bPMeCAR BCP to obtain perpendicular lamellae will be specified. As explained in the introduction chapter, neutral substrate and air interfaces are required to obtain perpendicular lamellae orientation. Therefore, identification of a neutral underlayer is an important first step for orientation control. For BCPs with SAP additive formulations to be implemented in fabrication processes, successful DSA (i.e., defect-free long range order) and the ability to selectively remove a BCP domain have to be demonstrated.

Table 1 presents a summary of the main experiments carried out, while Figure 16 shows the structure of PS-b-PMeCAR and a schematic of the process.

Table 1. Summary of experiments for orientation control of BCP thin film and DSA.

\begin{tabular}{|c|c|c|c|c|}
\hline Step & $\begin{array}{c}\text { Type of } \\
\text { experiments }\end{array}$ & Parameters & Values & Characterization \\
\hline $\begin{array}{l}\text { Neutral } \\
\text { Substrate } \\
\text { Interface } \\
\end{array}$ & $\begin{array}{l}\text { Spin coating of } \\
\text { PS-r-PMMA }\end{array}$ & $\begin{array}{l}\text { Variation of PS } \\
\text { composition }\end{array}$ & $0-32 \% \mathrm{PS}$ & $\begin{array}{c}\text { AFM (Step } \\
\text { height } \\
\text { measurements) }\end{array}$ \\
\hline \multirow{3}{*}{$\begin{array}{l}\text { Control of } \\
\text { Air } \\
\text { Interface }\end{array}$} & \multirow{3}{*}{$\begin{array}{l}\text { Spin coating of } \\
\text { PS-b- } \\
\text { PMeCAR + } \\
\text { SAP }\end{array}$} & SAP composition & $\begin{array}{c}\text { SAP 1 } \\
\text { SAP 2-40 } \\
\text { SAP 2-80 }\end{array}$ & \multirow{3}{*}{$\begin{array}{c}\text { AFM } \\
\text { GISAXS } \\
\text { XPS }\end{array}$} \\
\hline & & $\begin{array}{c}\text { SAP } \\
\text { concentration }\end{array}$ & $2-3$ wt $\%$ & \\
\hline & & Film thickness & $30-40 \mathrm{~nm}$ & \\
\hline \multirow{2}{*}{$\begin{array}{l}\text { Directed } \\
\text { Self } \\
\text { Assembly }\end{array}$} & \multirow[t]{2}{*}{ Graphoepitaxy } & Temperature & $\begin{array}{l}170{ }^{\circ} \mathrm{C} \\
200{ }^{\circ} \mathrm{C}\end{array}$ & \multirow{2}{*}{ SEM } \\
\hline & & Time & $\begin{array}{l}5 \text { min } \\
10 \text { min }\end{array}$ & \\
\hline
\end{tabular}


a.<smiles>CCC(C)CC(CCOC(=O)OCC(C)(COC(C)=O)C(=O)OC)c1ccccc1</smiles>

$\operatorname{PS}(12.5 \mathrm{k})-b-\operatorname{PMeCAR}(14.5 \mathrm{k}) \mathrm{Vf}_{\mathrm{PMeCAR}} \sim 0.48$

b.
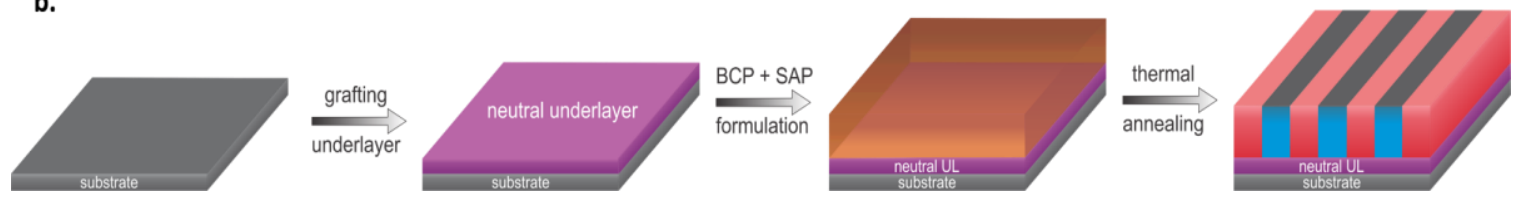

Figure 16. (a) The structure of the BCP, PS-b-PMeCAR. (b) A schematic of the experimental process, starting with identifying the neutral underlayer, varying the SAP composition and concentration to obtain perpendicular lamellae, and directed selfassembly to orient the lamellae in-plane.

To identify the neutral substrate, the substrate surface was modified using hydroxide terminated random copolymers, as described by Mansky et al. [24]. By varying the PS composition of the PS-r-PMMA used to modify the substrate surface, and then spin coating and annealing the PS-b-PMeCAR, step height features form. The step height features can be used to identify the neutral underlayer by measuring via AFM which PS composition forms $0.5 \mathrm{~L}_{0}$ step height features, as reported by Kim et al. [27].

For air interface control, different concentrations of three different SAP compositions were used to determine the amount of SAP additive needed to pull the more polar BCP component to the surface. Two different film thicknesses were compared as well in order to observe how the SAP worked in a thicker film since not all processes use the same film thickness. The AFM was used to observe the film surface and confirm the presence of perpendicular lamellae. 
A reactive ion etch (RIE) process and a sequential infiltration synthesis (SIS) process were applied to thin film samples with successful perpendicular orientation to show that they can be successfully used to selectively remove a BCP domain.

Demonstration that a domain can be selectively removed is important to show in order for SAP additives to be considered for patterning applications.

As stated in the introduction, along with out-of-plane orientation control, in-plane alignment is also important for the use of BCPs in patterning applications. For the DSA step, graphoepitaxy was attempted on a SAP additive containing BCP formulation that showed successful perpendicular orientation. However, because the initial annealing conditions used did not give satisfactory results, temperature and time were varied to improve the alignment [48]. To check the BCP alignment along the pre-pattern, SEM was used.

\subsection{Materials}

The PS-b-PMeCAR BCP, and SAP additives used were synthesized at IBM Research-Almaden. The properties of PS-b-PMeCAR are as follows: $\mathrm{Mn}_{\mathrm{PS}}=12,500$ $\mathrm{g} / \mathrm{mol}, \mathrm{Mn}_{\mathrm{PMeCAR}}=14,500 \mathrm{~g} / \mathrm{mol}$, volume fraction of PMeCAR block, $\mathrm{Vf}_{\mathrm{PMeCAR}} \sim 0.48$, and PDI $=1.02$.

The PS-r-PMMA random copolymers are hydroxyl-end functionalized in order to graft onto the silicon substrate and were used as underlayers. The PS-r-PMMA solutions consisting of 0 and $20 \%$ styrene were provided by EMD Performance Materials Corporation (AZEMBLY ${ }^{\mathrm{TM}}$ NLD-303 and AZEMBLY ${ }^{\mathrm{TM}}$ NLD-328J, respectively). The PS-r-PMMA solution consisting of $32 \%$ styrene was synthesized at IBM. 


\subsection{Methods}

\subsubsection{Preparation of Underlayer Solution}

For the $32 \%$ styrene underlayer solution prepared on site at IBM, the PS-r-PMMA copolymer was obtained in powder form. The copolymer solution was made by dissolving the powder PS-r-PMMA in PGMEA, to make a 1.0 wt \% with a 95:5 weight ratio of PS-r-PMMA to PNBT. To remove particles, the underlayer solution was filtered through a $0.2 \mu \mathrm{m}$ nylon filter. The 0 and $20 \%$ styrene solutions were used as received, and did not require filtering, as they were already filtered.

\subsubsection{Preparation of BCP solution and SAP formulations}

The PS-b-PMeCAR, was obtained in powder form, and was synthesized using the method described by Vora et al. for ring opening polymerization of carbonates from PS$\mathrm{OH}$ [32]. The powder was dissolved in PGMEA, to create a $1.2 \mathrm{wt} \%$ solution. To prepare the BCP with SAP additive formulations, a $1.2 \mathrm{wt} \%$ stock solution of the SAP in PGMEA was used, and 2-3 wt \% of SAP stock solution was added to the BCP stock solution. Once fully dissolved, the solution was passed through a $0.2 \mu \mathrm{m}$ nylon filter to remove particles.

\subsubsection{Identification of Neutral Underlayer}

Hydroxyl terminated PS-r-PMMA solutions of varying ratios of styrene and methyl methacrylate were grafted onto bare silicon by spin coating at $2000 \mathrm{rpm}$ for 30 seconds and subsequently annealed. For the underlayer solutions prepared at IBM, the substrate was annealed at $200{ }^{\circ} \mathrm{C}$ for 3 minutes. For the underlayer solutions provided by 
EMD Performance Materials Corporation, the substrates were annealed at $250{ }^{\circ} \mathrm{C}$ for 2 minutes.

The substrates were rinsed with PGMEA to remove any unreacted material. This was done by spin coating the PGMEA on the substrates at $2000 \mathrm{rpm}$ for 30 seconds. Afterwards, the BCP solution without any SAP was spin coated at $2000 \mathrm{rpm}$ for 30 seconds. The samples were annealed at $170{ }^{\circ} \mathrm{C}$ for 5 minutes which is well above the $\mathrm{T}_{\mathrm{g}}$ temperature of PS (which has the higher $\mathrm{T}_{\mathrm{g}}$ of the two $\mathrm{BCP}$ components at about $100{ }^{\circ} \mathrm{C}$ ). The thin films were characterized by AFM to measure the step height of the islands and holes. Using the method proposed by Kim et al., an underlayer that showed a step height of $0.5 \mathrm{~L}_{0}$ was selected as the neutral underlayer [27].

\subsubsection{Thin Film Sample Preparation}

The neutral underlayer was applied onto the silicon substrate under the same conditions described above. After rinsing with PGMEA to remove any unreacted material, the BCP with SAP additive formulation was spin coated between 1500-2000 rpm, depending on desired film thickness, for 30 seconds. The sample was annealed at $170{ }^{\circ} \mathrm{C}$ for 5 minutes. The surface topography was analyzed using AFM. The AFM was used to determine orientation of the lamellae. Additional analysis of the thin film was done using GISAXS and XPS.

\subsubsection{Preparation of Graphoepitaxy Pre-pattern}

AZEMBLYTM NLD-328J (20\% PS) was spin coated at $2000 \mathrm{rpm}$ for 30 seconds on a silicon wafer substrate stack comprised of a silicon wafer bottom layer coated with $\sim 30 \mathrm{~nm}$ thick amorphous carbon layer and $10 \mathrm{~nm}$ thick silicon nitride $\left(\operatorname{SiN}_{\mathrm{x}}\right)$ layer. After 
forming the thin film, the coated wafer was baked at $250{ }^{\circ} \mathrm{C}$ for 2 minutes and cooled to room temperature. The underlayer was then given a solvent rinse by casting PGMEA on top of the coated wafer, letting the solvent puddle for 30 seconds, and spin drying the treated wafer at $2000 \mathrm{rpm}$ for 30 seconds. The final film thickness of the underlayer was $10 \mathrm{~nm}$ after the solvent rinse. Next, a $60 \mathrm{~nm}$ thick layer of a commercial $193 \mathrm{~nm}$ negative-tone photoresist (JSR ARF7210JN-8) was disposed on this underlayer coated substrate followed by post application bake at $80{ }^{\circ} \mathrm{C}$ for 60 seconds. The photoresist layer was then exposed using a $193 \mathrm{~nm}$ immersion interference tool (IBM NEMO) with fixed dose of $4.67 \mathrm{~mJ}$, baked at $95^{\circ} \mathrm{C}$ for 60 seconds, and developed for 60 seconds with 2-heptanone developer. The resulting $200 \mathrm{~nm}$ pitch patterned photoresist layer was then hard baked at $200{ }^{\circ} \mathrm{C}$ for 3 minutes prior to coating a block copolymer formulation.

\subsubsection{Preparation of Graphoepitaxy DSA Samples}

PS-b-PMeCAR BCP was dissolved in PGMEA to form a $0.8 \mathrm{wt} \%$ stock solution based on total weight of the solution. A separate $0.8 \mathrm{wt} \%$ stock solution of the selected SAP in PGMEA was prepared. The desired amount of SAP stock solution was added to the BCP solution and stirred to form a homogeneous solution. The BCP with SAP additive formulation was passed through a $0.2 \mu \mathrm{m}$ nylon filter to remove particles.

The formulation was spin coated onto the pre-patterned substrate described in the previous section at $2000 \mathrm{rpm}$ for 30 seconds. It was subsequently thermal annealed at the desired temperature and time, and immediately cooled to room temperature. Alignment and defectivity inside the guiding pre-pattern trenches was determined using top-down SEM. 


\subsubsection{SIS Process}

A thin film of the BCP was placed in a Cambridge NanoTech Fiji F200 at $75^{\circ} \mathrm{C}$. Trimethyl aluminum (TMA) was admitted into the reactor at a pressure of 70 Torr for 5 minutes. The chamber was evacuated to a pressure below 20 mTorr to remove excess TMA and byproducts. Water was then introduced at a pressure of 70 Torr for 5 minutes and then purged to complete the cycle. The process was repeated for 7 cycles.

\subsubsection{Etch Process}

Etching experiments without SIS were done on a trilayer stack consisting of a silicon substrate, followed by $\alpha$-carbon $(30 \mathrm{~nm}), \mathrm{SiNx}(10 \mathrm{~nm})$, and the underlayer. To remove the PMeCAR but preserve the PS, an $\mathrm{O}_{2}$ etch was used in a Lam Research 4520 XLe dielectric etcher. The following parameters were used: $7 \mathrm{sec}, 300 \mathrm{mTorr}, \mathrm{O}_{2}$ flow rate of $136 \mathrm{sccm}$, lower electrode power $50 \mathrm{~W}$, upper electrode power $0 \mathrm{~W}$, and gap between electrodes $2.25 \mathrm{~cm}$. To remove $\mathrm{SiN}_{\mathrm{X}}$, a mixture of $\mathrm{CF}_{4}$ and $\mathrm{CHF}_{3}$ was used by a Plasma Therm Versaline ICP etcher. The following parameters were used: $13 \mathrm{sec}, 4$ mTorr, $\mathrm{CF}_{4}$ flow rate of $20 \mathrm{sccm}, \mathrm{CHF}_{3}$ flow rate of $15 \mathrm{sccm}$, bias power $50 \mathrm{~W}$, and coil power $100 \mathrm{~W}$. To remove the $\alpha$-carbon, a mixture of $\mathrm{O}_{2}$ and $\mathrm{N}_{2}$ was used in the same etcher as for $\mathrm{SiN}_{\mathrm{X}}$. The following parameters were used for this step: $30 \mathrm{sec}, 4 \mathrm{mTorr}$, $\mathrm{O}_{2}$ flow rate $2 \mathrm{sccm}, \mathrm{N}_{2}$ flow rate $40 \mathrm{sccm}$, bias power $100 \mathrm{~W}$, and coil power $200 \mathrm{~W}$.

The etch process for the SIS modified sample was done with a Lam Research 4520 XLe dielectric etch tool. To remove the PS domain, the following parameters were used: $10 \mathrm{sec}, 500 \mathrm{mTorr}, \mathrm{O} 2$ flow rate of $136 \mathrm{sccm}$, lower electrode power $50 \mathrm{~W}$, upper 
electrode power $0 \mathrm{~W}$, and gap between electrodes $2.25 \mathrm{~cm}$. All of the samples post etch process were imaged using a Leo Gemini SEM to characterize the morphology.

\subsection{Instrumentation}

\subsubsection{AFM}

A Digital Instruments 3100 AFM operated in tapping mode in air using a Si cantilever with a $1 \mathrm{~N} / \mathrm{m}$ spring constant was used to analyze the surface topography of the BCP thin films. The typical scan rate used was $1 \mathrm{~Hz}$, with image sizes varying from $2 \mu \mathrm{m} \times 2 \mu \mathrm{m}$ to $5 \mu \mathrm{m} \times 5 \mu \mathrm{m}$. The $5 \mu \mathrm{m} \times 5 \mu \mathrm{m}$ scan size was used to measure step height differences to identify the neutral underlayer, while the $2 \mu \mathrm{m} \times 2 \mu \mathrm{m}$ scan sizes were used to determine the orientation of the lamellae and to measure the pitch of the BCP. The step height measurements were done using the section cut, and the pitch measurements were done using the section and power spectral density features on the Nanoscope Analysis v. 1.5 AFM software by Bruker.

\subsubsection{SEM}

A Leo Gemini scanning electron microscope (SEM) at $10.0 \mathrm{kV}$ electron charge was used to measure the DSA samples. For top-down images of the samples throughout the etch process, a 100k magnification was used. A 200k magnification was used to take the images of the post-etch cross-sections, graphoepitaxy pre-pattern, and DSA samples.

\subsubsection{GISAXS}

Grazing incidence small angle X-ray scattering (GISAXS) was used to analyze lamellae orientation over a larger area and throughout the film of a sample. The GISAXS data was collected at the Advanced Light Source (ALS) at Lawrence Berkeley National 
Laboratory (LBNL) at beam line 7.3.3 [49]. The incident X-ray energy was $10 \mathrm{keV}$ and the sample to detector distance was $4 \mathrm{~m}$. Scattered X-rays were collected using a Pilatus 2M detector. Data was normalized for incoming x-ray intensity, film thickness and wafer

size, averaged, and integrated along $\mathrm{q}_{\mathrm{x}}=0.028 \AA^{-1}$ using the IRENA package, developed by I. Ilavsky et al. [50]. The scattering profiles were analyzed by fitting a series of Voigt peaks and an exponential background to the 1D data. The periodicity was calculated from the $1^{\text {st }}$ order Bragg peak by $d=2 \pi / q$, where $d$ is the periodicity and $q$ is the scattering wave vector.

\subsubsection{XPS}

X-ray photoelectron spectroscopy (XPS) analysis was done on a Physical Electronics Quantum ESCA Microprobe, using a monochromated $\mathrm{AlK}_{\mathrm{a}} \mathrm{X}$-ray source at $187.85 \mathrm{eV}, 200 \mu \mathrm{m}$ spot size throughout, and charge neutralization. High resolution spectra were taken at three different angles for depth profiling: $15^{\circ}(47 \mathrm{eV} \mathrm{PE}, 0.4$ $\mathrm{eV} / \mathrm{step}$, electron escape depth $~ 3 \mathrm{~nm}$ ), $45^{\circ}$ (59 eV PE, $0.4 \mathrm{eV} / \mathrm{step}$, electron escape depth $\sim 8 \mathrm{~nm}$ ), and $80^{\circ}$ (24 eV PE, $0.2 \mathrm{eV} / \mathrm{step}$, electron escape depth $~ 12 \mathrm{~nm}$ ). 


\section{CHAPTER FIVE}

\section{RESULTS AND DISCUSSION}

The results obtained for self-assembly and DSA using a high- $\chi$ BCP based on a polycarbonate platform are discussed in this chapter. The discussion is divided into sections in sequential order, starting with identification of a neutral underlayer, followed by orientation control using different SAP additives, etch and SIS results, and then alignment using graphoepitaxy DSA.

\subsection{Random Copolymer as Neutral Underlayer}

As the first step to control the orientation of the PS-b-PMeCAR lamellae, a neutral underlayer was identified. For thermally annealed systems, a non-preferential substrate interface can be obtained by grafting random copolymers onto the substrate. For PS-b-PMeCAR, hydroxyl end-functional PS-r-PMMA copolymer brushes with varying amounts of PS were chosen to modify the substrate interface. To identify which composition is neutral for the $\mathrm{BCP}$, a qualitative method previously presented by Kim et al. was used, in which the formation of $0.5 \mathrm{~L}_{0}$ island or hole step height features appear when only one interface is neutral [27]. For PS-b-PMeCAR, polystyrene has the lower surface energy of the two BCP components, and therefore is expected to be the only material at the air-BCP interface, making the air-BCP interface non-neutral [32]. If the random copolymer brush is non-neutral, then $1 \mathrm{~L}_{0}$ step height features form, whereas $0.5 \mathrm{~L}_{0}$ step height features form only if the random copolymer brush is neutral. The step heights were measured by AFM. Using a PMMA brush (0\% S), the AFM image shown in Figure $17 \mathrm{a}$, shows hole morphology with approximately $1 \mathrm{~L}_{0}$ step height $(\mathrm{L}=18.9 \mathrm{~nm})$. 
This sample exhibits asymmetric wetting, with the PMeCAR block wetting the BCP substrate interface and PS wetting the air interface. In contrast, when a $32 \%$ S containing underlayer was used, Figure $17 \mathrm{c}$ shows island morphology with approximately $1 \mathrm{~L}_{0}$ step height $(\mathrm{L}=19.3 \mathrm{~nm})$, as expected for symmetric wetting with PS at the bottom and top interface. The transition from hole to island morphology indicates that the neutral composition lies in between the two compositions. Indeed, by using a $20 \% \mathrm{~S}$ containing underlayer, approximately $0.5 \mathrm{~L}_{0}(\mathrm{~L}=9.0 \mathrm{~nm})$ step height hole morphology was seen, as shown in Figure 17b, identifying this underlayer as neutral.

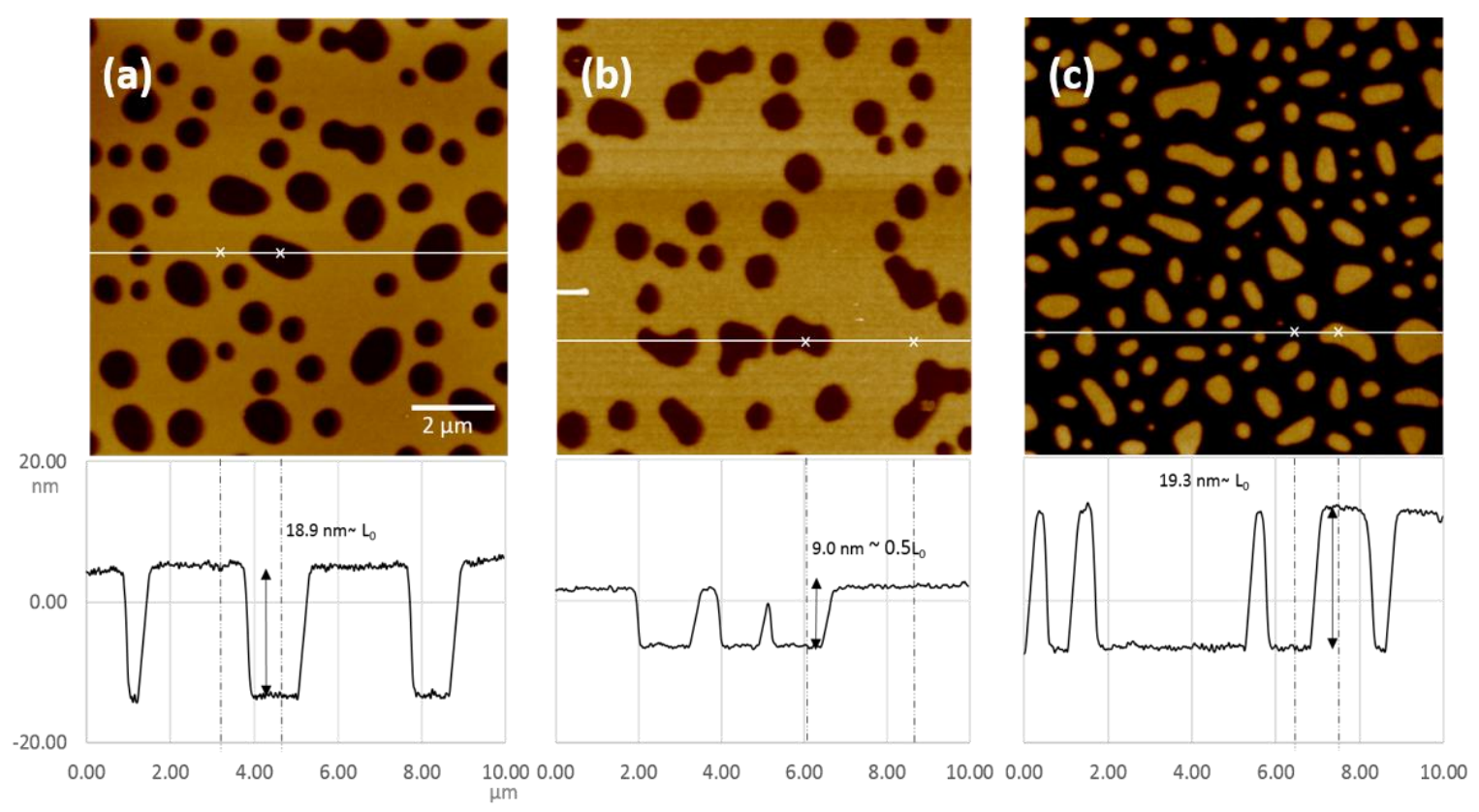

Figure 17. Atomic force microscope topographic images of (a) asymmetrical wetting showing holes with $1 \mathrm{~L}_{0}$ step height on PMeCAR preferential UL $(0 \% \mathrm{~S})$, (b) $0.5 \mathrm{~L}_{0}$ step height on neutral UL $\left(20 \%\right.$ S), and (c) symmetrical wetting showing islands with $1 \mathrm{~L}_{0}$ step height on PS preferential UL (32\% S), with corresponding height graphs of a surface section to measure height differences. 


\subsection{Air Interface Control}

Following the successful identification of $20 \% \mathrm{~S}$ as the neutral underlayer, formulations of PS-b-PMeCAR with different SAP compositions and concentrations were prepared for orientation control at the air interface. In general, fluorinated groups are surface active, and therefore have a lower surface energy than polystyrene [38]. By using polymers containing fluorinated groups, it was postulated that these SAPs could enable orientation control at the BCP-air interface through two key design parameters. First, the SAP has a lower surface energy than either of the individual BCP components, PS or PMeCAR in this case. Second, the SAP has a preferential interaction with the more polar, higher surface energy block of the BCP. The combined design parameters in the SAP should help in balancing the surface energies of the two BCP components at the BCP-air interface by preferentially interacting with the polar component and bringing it to the air interface. In this section, the results of the effects of the different SAP compositions and concentrations on orientation are presented.

The first SAP composition studied, SAP 1, was a homopolymer, poly(hexafluoro alcohol styrene) (PHFAS), shown in Figure 18. For this SAP composition, the $\mathrm{CF}_{3}$ groups on the styrene are meant to give the SAP a lower surface energy than either BCP components, fulfilling the first design parameter. The hydroxyl group acts as a hydrogen bond donor, and preferentially interacts with the carbonyl group of the methyl carbonate in the BCP, fulfilling the second design parameter [47]. 


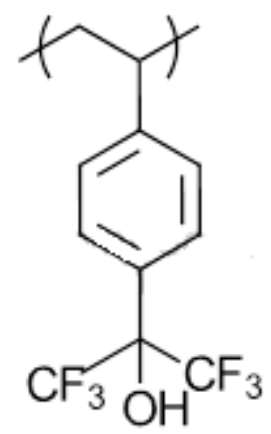

Figure 18. Structure of PHFAS (SAP 1).

Two different concentrations of SAP 1 ( 2 and 3 wt \%) were added to the BCP solution and spin coated onto silicon substrates with a neutral underlayer of $20 \% \mathrm{~S}$, and characterized by AFM, shown in Figure 19. Both concentrations resulted in perpendicular lamellae orientation, and the distinct features shown in the phase AFM images demonstrate that two different compositions are at the surface (PS and PMeCAR). The pitch of the BCP was measured to be approximately $20.7 \mathrm{~nm}$, which is within reasonable agreement with the pitch measured using GISAXS (19.87 nm). However, 2 wt $\%$ SAP 1 contains areas in which parallel lamellae form, as shown in Figure 19a. These defects are undesired for later pattern transfer applications into the silicon substrate. On the other hand, when 3 wt $\%$ SAP 1 was used, perpendicular lamellae were seen to form uniformly over a $2 \mu \mathrm{m}$ x $2 \mu \mathrm{m}$ area coverage, as shown in Figure 19b. Based on this observation, it is possible that at $2 \mathrm{wt} \%$ there is not enough SAP 1 to uniformly bring the methyl carbonate groups to the air interface. These results show that despite having a surface active group present, if the group is not sufficiently surface active, then the SAP will be incapable of fully controlling lamellae orientation. Either a 
higher concentration of SAP 1 is needed, as shown by the need to use $3 \mathrm{wt} \%$, or a more surface active group is necessary for proper orientation control.
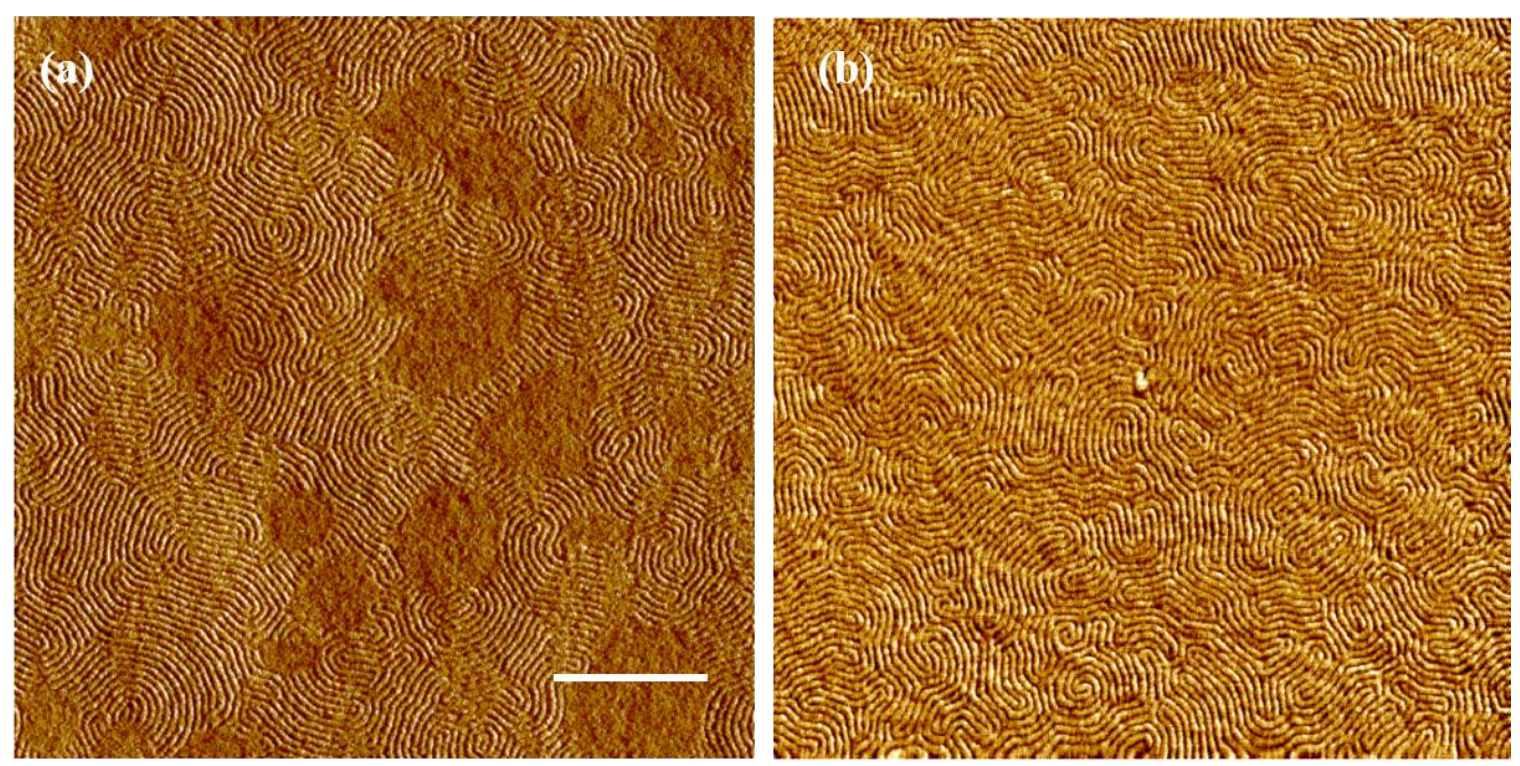

Figure 19. Atomic force microscope phase images of PS-b-PMeCAR + SAP 1 on a modified neutral substrate with (a) $2 \mathrm{wt} \%$ and (b) $3 \mathrm{wt} \%$ SAP 1 . Scale bar: $400 \mathrm{~nm}$.

The next SAP composition studied was a random copolymer consisting of HFAS (same as SAP 1) and pentafluorostyrene (PFS), referred to as SAP 2, shown in Figure 20. The addition of PFS (a fluorinated styrene) further lowers the surface energy of SAP 2 in comparison to SAP 1, making SAP 2 more surface active. The hydroxyl group on the HFAS group still acts as a hydrogen bond donor to preferentially interact with the carbonyl group in the PMeCAR block. With this, the two SAP design parameters of having a lower surface energy than either of the $\mathrm{BCP}$ components and selective interaction with the more polar block (PMeCAR), are fulfilled. 


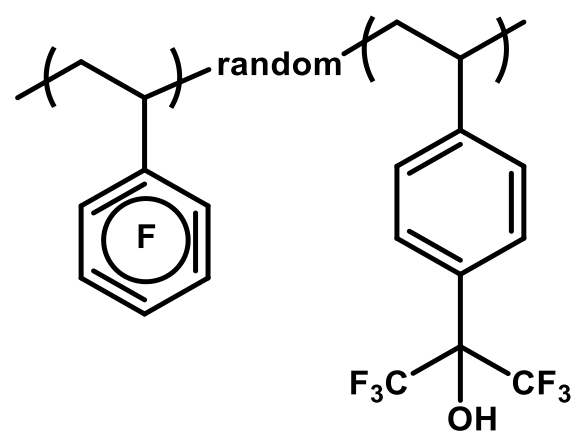

Figure 20. Structure of SAP 2, PHFAS-r-PPFS.

Because SAP 2 is a random copolymer containing two different components, a study varying the mole ratio of PFS to HFAS was done to better understand the effect of surface activity. The two different ratios studied were 40:60 (SAP 2-40) and 80:20 (SAP 2-80). By increasing the amount of PFS, the surface energy of the SAP is further reduced, so SAP 2-80 is more surface active than either SAP 1 or SAP 2-40.

The first SAP ratio studied was SAP 2-40. Again, 2 and $3 \mathrm{wt} \%$ concentrations were added to PS-b-PMeCAR solutions, spin coated onto a silicon substrate with a neutral underlayer, and characterized by AFM, as shown in Figure 21. Both concentrations showed perpendicular lamellae formation, with distinct phases seen in the AFM phase images, indicating the presence of both block components on the surface. The pitch in this case was measured to be $20.8 \mathrm{~nm}$, similar to the pitch using SAP 1 (20.7 nm). However, at a 3 wt $\%$ concentration, defects can be seen through the surface in the form of parallel lamellae areas, shown in Figure 21b. At a 2 wt \% concentration, the AFM phase image shows full coverage of perpendicular lamellae in a $2 \mu \mathrm{m}$ x $2 \mu \mathrm{m}$ area, shown in Figure 21a. For the SAP 2-40, a 3 wt \% concentration appears to be too high. 
Unlike the results seen using SAP 1, where a higher amount of SAP 1 was needed for orientation control, for SAP 2-40, which is more surface active than SAP 1, a higher amount of SAP 2-40 becomes too surface active.
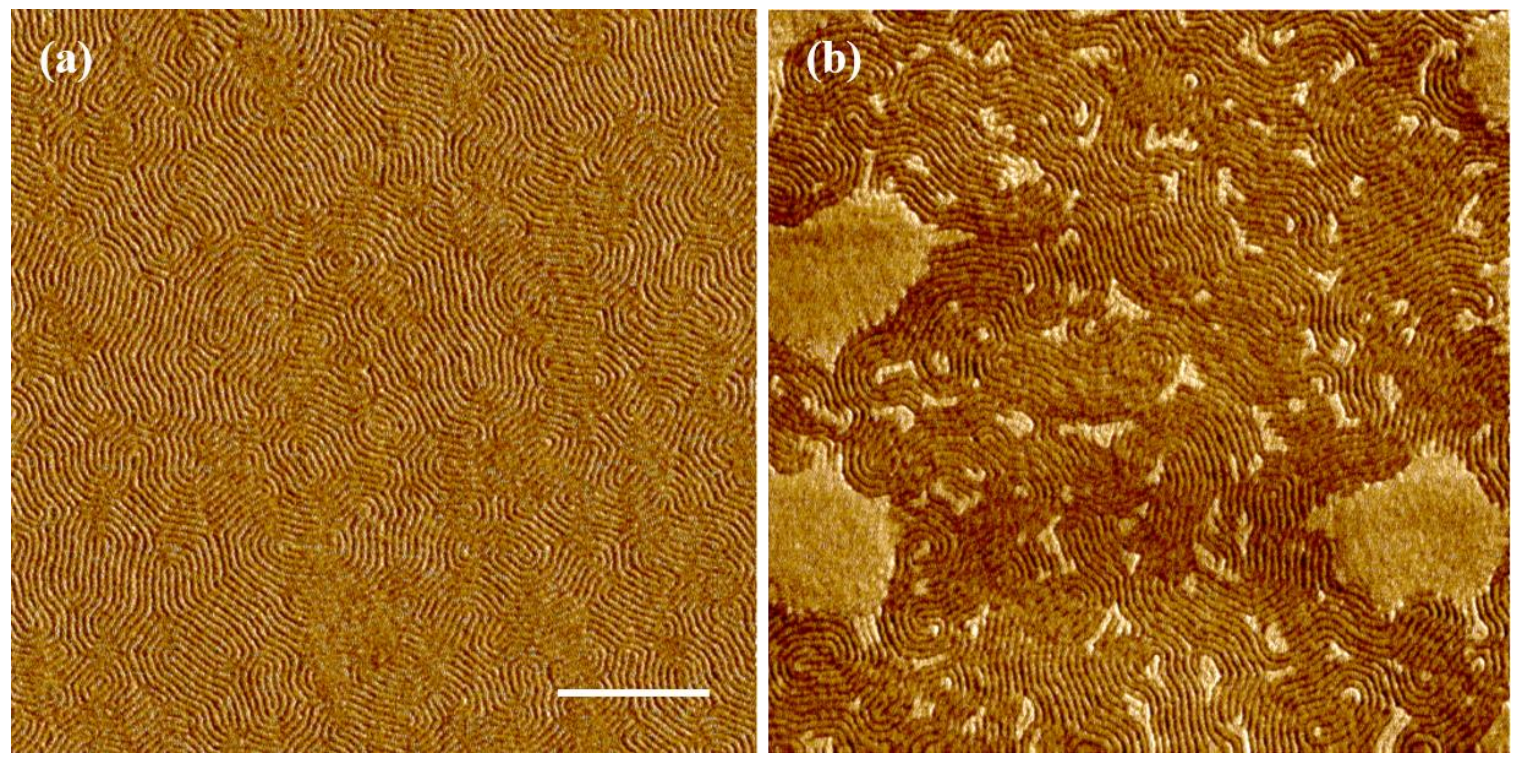

Figure 21. Atomic force microscope phase images of PS-b-PMeCAR + SAP 2-40 with (a) $2 \mathrm{wt} \%$ and (b) $3 \mathrm{wt} \%$ SAP 2-40: Scale bar: $400 \mathrm{~nm}$.

Because of the higher surface activity, the SAP 2-40 segregates to the air interface and pulls the hydrogen bonded methyl carbonate phase towards the surface as well to form parallel lamellae. Therefore, a balance of surface activity between the PS and the SAP modified carbonate must be reached for orientation control to occur.

A film thickness study was done as well on a 2 wt $\%$ SAP 2-40 formulation in order to determine how the SAP acted in thicker films. Film thicknesses of approximately 33 and $43 \mathrm{~nm}$ were prepared and characterized via AFM. For a thicker film, defects in the form of parallel lamellae were visible across the surface, as shown in Figure 22b. This could be a result of the SAP becoming trapped in the bulk of the thin 
film due to the limiting surface activity of the SAP, or the molecular weight of the additive being kinetically trapped.
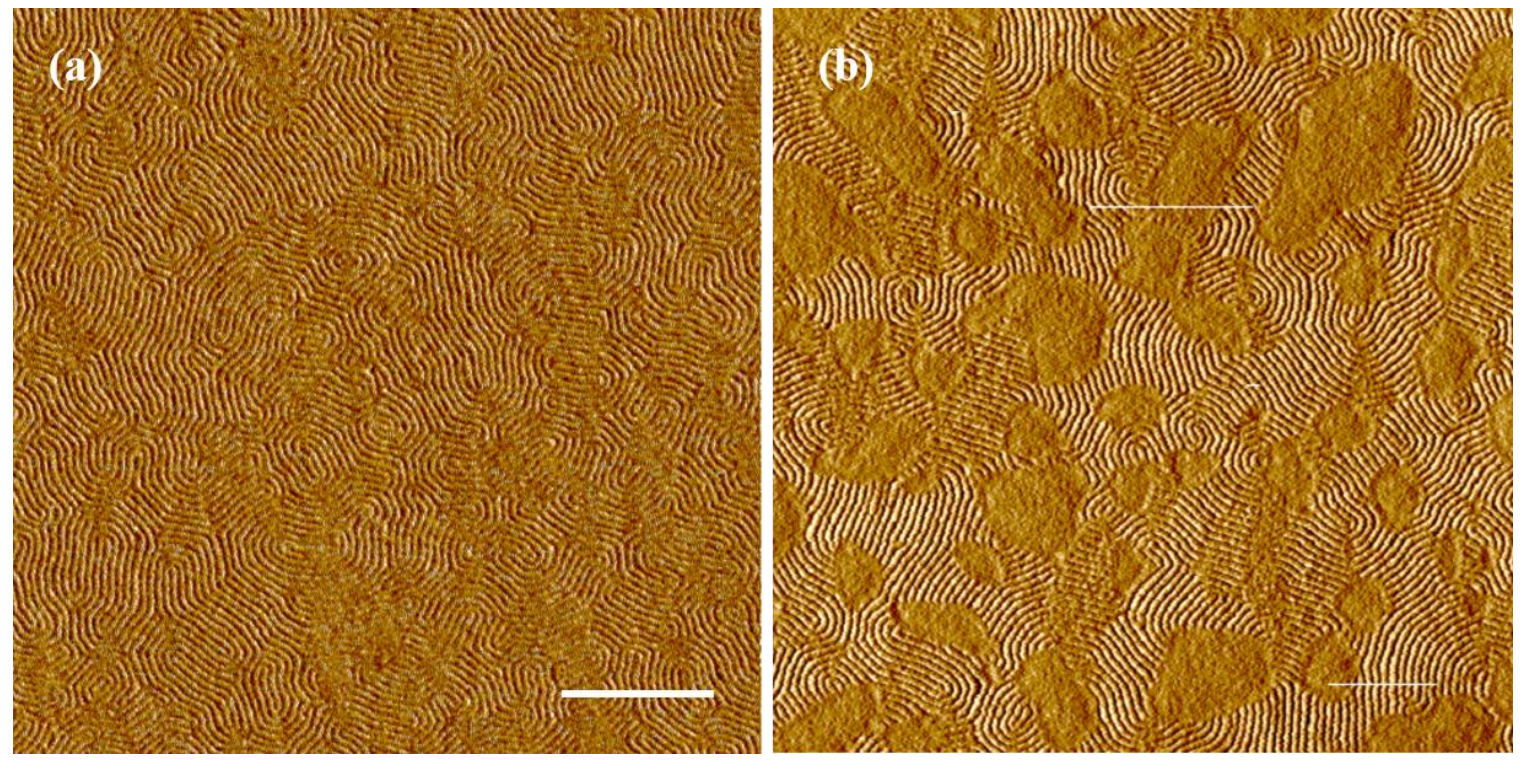

Figure 22. Atomic force microscope phase images of PS-b-PMeCAR $+2 \%$ SAP 2-40 at film thicknesses (a) $33 \mathrm{~nm}$ and (b) $43 \mathrm{~nm}$. Scale bar: $400 \mathrm{~nm}$.

For SAP 2-80, which has a higher surface activity than SAP 2-40 due to the higher content of PFS, concentration studies using 2 and $3 \mathrm{wt} \%$ and characterization by AFM were done as well. The results, shown in Figure 23, are similar to those of SAP 240. At $2 \mathrm{wt} \%$, perpendicular lamellae are seen across a $2 \mu \mathrm{m} \times 2 \mu \mathrm{m}$ AFM phase image shown in Figure 23a, while at 3 wt \%, defects in the form of parallel lamellae are seen across the surface, as seen in Figure $23 \mathrm{~b}$. Due to SAP 2-80 being so similar to SAP 2-40 in design, with the difference of a higher surface activity, these results are unsurprising.

The AFM images of the thickness study for 2 wt \% SAP 2-80, with film thicknesses of 33 and $43 \mathrm{~nm}$, are shown in Figure 24. With SAP 2-80, defects were no longer present on the thicker film like they were when using SAP 2-40. The results 
between the SAP 2-40 and SAP 2-80 thickness studies imply that by using a more surface active SAP, less of the SAP becomes trapped in the thin film, allowing for perpendicular lamellae to form in thicker films.
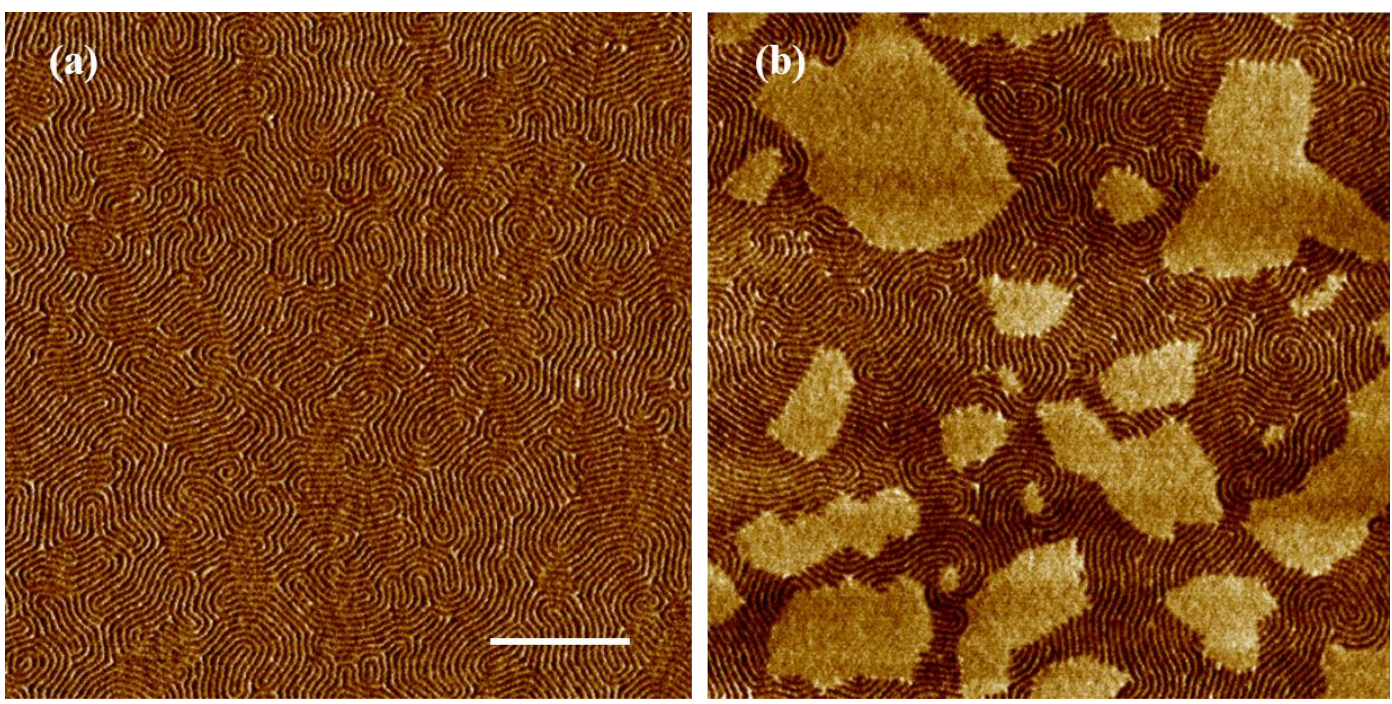

Figure 23. Atomic force microscope phase images of PS-b-PMeCAR + SAP 2-80 with (a) $2 \mathrm{wt} \%$ SAP 2-80, showing no defects (b) and $3 \mathrm{wt} \%$ SAP 2-80, with flat areas. Scale bar: $400 \mathrm{~nm}$.
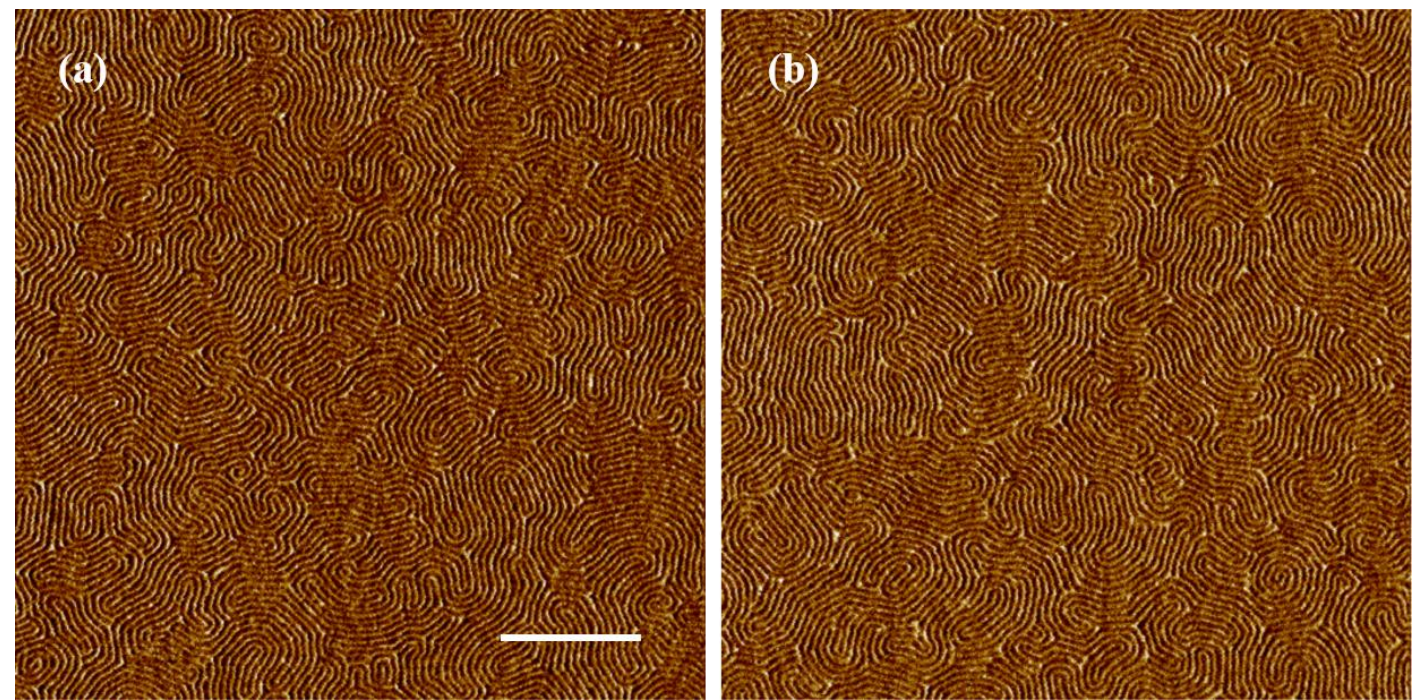

Figure 24. Atomic force microscope phase images of PS-b-PMeCAR +2 wt $\%$ SAP 280 at different film thicknesses (a) $33 \mathrm{~nm}$ and (b) $43 \mathrm{~nm}$. Scale bar: $400 \mathrm{~nm}$. 
To further confirm that the addition of SAP to the BCP enables perpendicular lamellae on a larger scale and throughout the film, GISAXS analysis was done on samples containing 3 wt $\%$ SAP 1, 2 wt $\%$ SAP 2-40, 2 wt $\%$ SAP 2-80, and a control sample with no SAP. The in-plane scattering profiles are shown in Figure 25. For the sample containing no SAP, no high intensity peaks are observed, with only a single weak $1^{\text {st }}$ order Bragg peak present. The lack of high intensity peaks and weak $1^{\text {st }}$ order Bragg peak signal indicate the lack of perpendicular lamellae in the film, with the majority of the lamellae being parallel to the substrate, which is in agreement with the AFM results shown earlier.

With the addition of the different SAP additive compositions, the in-plane scattering profiles were vastly different from the one without the SAP additive. All the thin film samples containing SAP display a much higher intensity $1^{\text {st }}$ order Bragg peak than the thin film without SAP, indicating a stronger presence of perpendicular lamellae in the films. Moreover, $2^{\text {nd }}$ and $3^{\text {rd }}$ order peaks formed as well, further demonstrating the presence of perpendicular lamellae within and throughout the film. Additionally, the positions of the three peaks form a ratio of 1:2:3, which is typical for lamellae structures [51]. The lower intensity of the $2^{\text {nd }}$ order peak in comparison to the $3^{\text {rd }}$ order peak is indicative of the two $\mathrm{BCP}$ components being present in equal amounts. The nearest neighbor spacing of the $\mathrm{BCP}$ (the pitch) was determined from the $1^{\text {st }}$ order Bragg peak by $\mathrm{d}=2 \pi / \mathrm{q}$ and was found to be $19.87 \mathrm{~nm}$. 


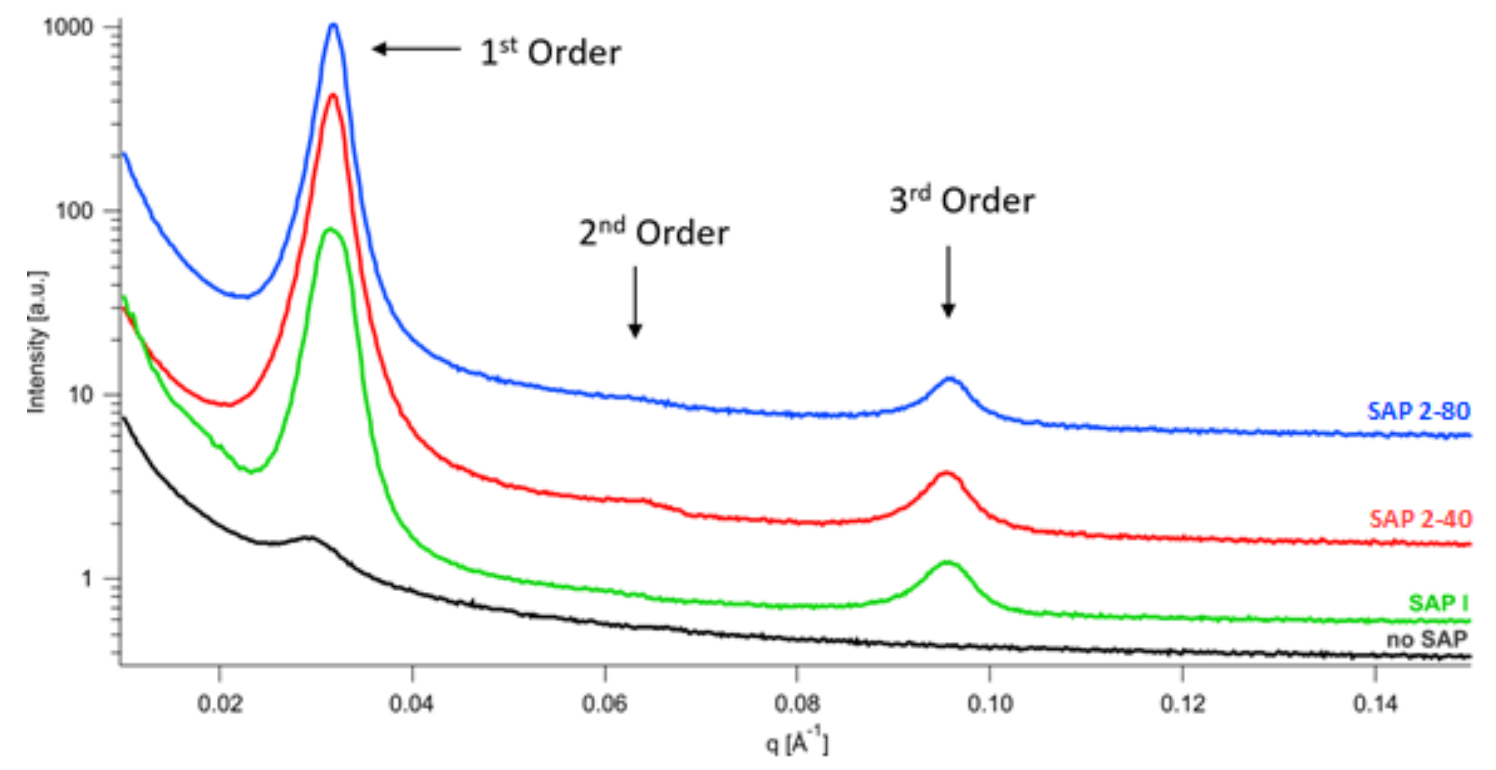

Figure 25. Plots of GISAXS in-plane scattering profiles for no SAP, SAP 1, SAP 2-40, and SAP 2-80. The sample with no SAP displays a weak $1^{\text {st }}$ order Bragg peak, indicating lack of perpendicular lamellae. Samples with SAP display a stronger $1^{\text {st }}$ order Bragg peak, and the presence of $2^{\text {nd }}$ and $3^{\text {rd }}$ order peaks in a 1:2:3 peak position ratio, typical for lamellae structures.

The purpose of the SAP is to pull the more polar component of a $\mathrm{BCP}$ to the surface of the film, and so the SAP is expected to be found at the surface. To confirm the location of the SAP additive in the film, X-ray photoelectron spectroscopy (XPS) was used to analyze the fluorine presence in the film as a characteristic element of the SAP. The fluorine presence was analyzed at different angles $\left(15^{\circ}, 45^{\circ}\right.$, and $\left.80^{\circ}\right)$. Each angle has a different electron escape depth $(\sim 3,8$, and $12 \mathrm{~nm}$, respectively). As the film thickness of the three samples was approximately $30 \mathrm{~nm}$, full depth profiling was not possible. Figure 26 plots the fluorine content in a sample with no SAP and a sample with 2 wt \% SAP 2-40. As expected, no fluorine was detected in the control sample with no SAP. For the sample with 2 wt $\%$ SAP $2-40$, at $15^{\circ}$, a clear peak is visible, indicating the presence of fluorine. At $45^{\circ}$, the fluorine peak is much more intense, and at $80^{\circ}$, the peak 
intensity increases, but is still very similar to the peak at $45^{\circ}$. As $99 \%$ of the detected electrons arise from within the first $3 \mathrm{~nm}$ for $15^{\circ}$ measurements, from within the first 8 $\mathrm{nm}$ for $45^{\circ}$, and $12 \mathrm{~nm}$ for $80^{\circ}$ measurements, it can be concluded that most of the fluorine is within the first $8 \mathrm{~nm}$ of the film.

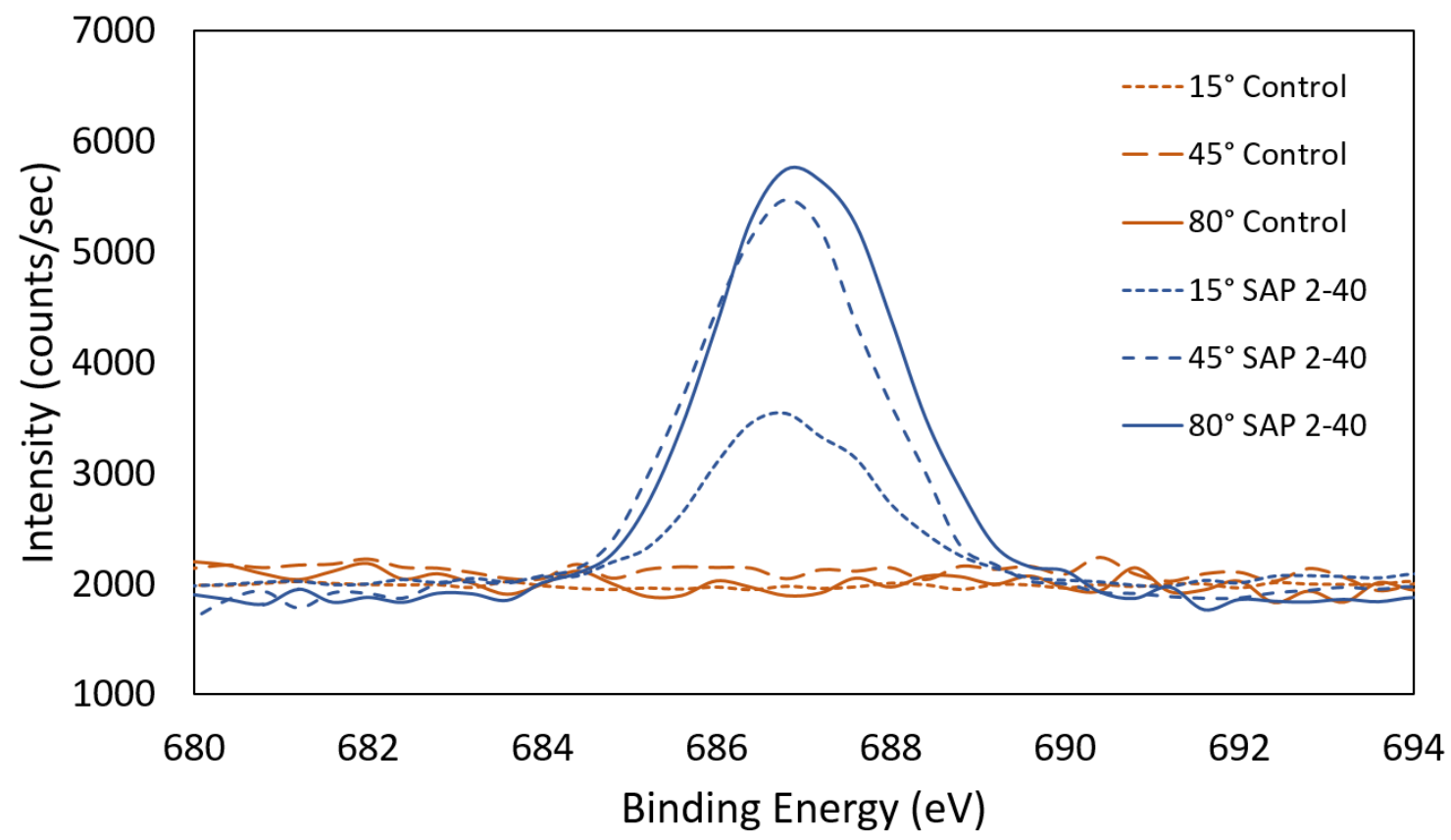

Figure 26. X-ray photoelectron spectra at the fluorine edge showing fluorine content at different detection depths in a control sample with no SAP and a sample with 2 wt \% SAP 2-40.

The oxygen profiles, shown in Figure 27, were consistent with the proposed morphology. For the control sample with no SAP additive, no oxygen was found within the first $3 \mathrm{~nm}$ of the film. Polystyrene does not contain oxygen, and so this confirms the presence of PS as a parallel layer at the surface of the film. Increasing the angle, and therefore probing deeper into the film, the oxygen signal appears at $45^{\circ}$ and becomes stronger at $80^{\circ}$. At $80^{\circ}$, the signal comes from the first $12 \mathrm{~nm}$ of the film, at which point 
electrons from the oxygen in the PMeCAR domain can be detected, making up the majority of the signal at $80^{\circ}$. These results are all consistent with parallel lamellae orientation.

For the sample with 2 wt \% SAP 2-40, the oxygen profile, shown in Figure 27, is consistent with a perpendicular lamellae morphology. At a $3 \mathrm{~nm}$ depth detection, oxygen is present, and as detection arises from deeper within the film, the oxygen signal steadily increases since the carbonate phase is present equally through the film because of the perpendicular orientation.

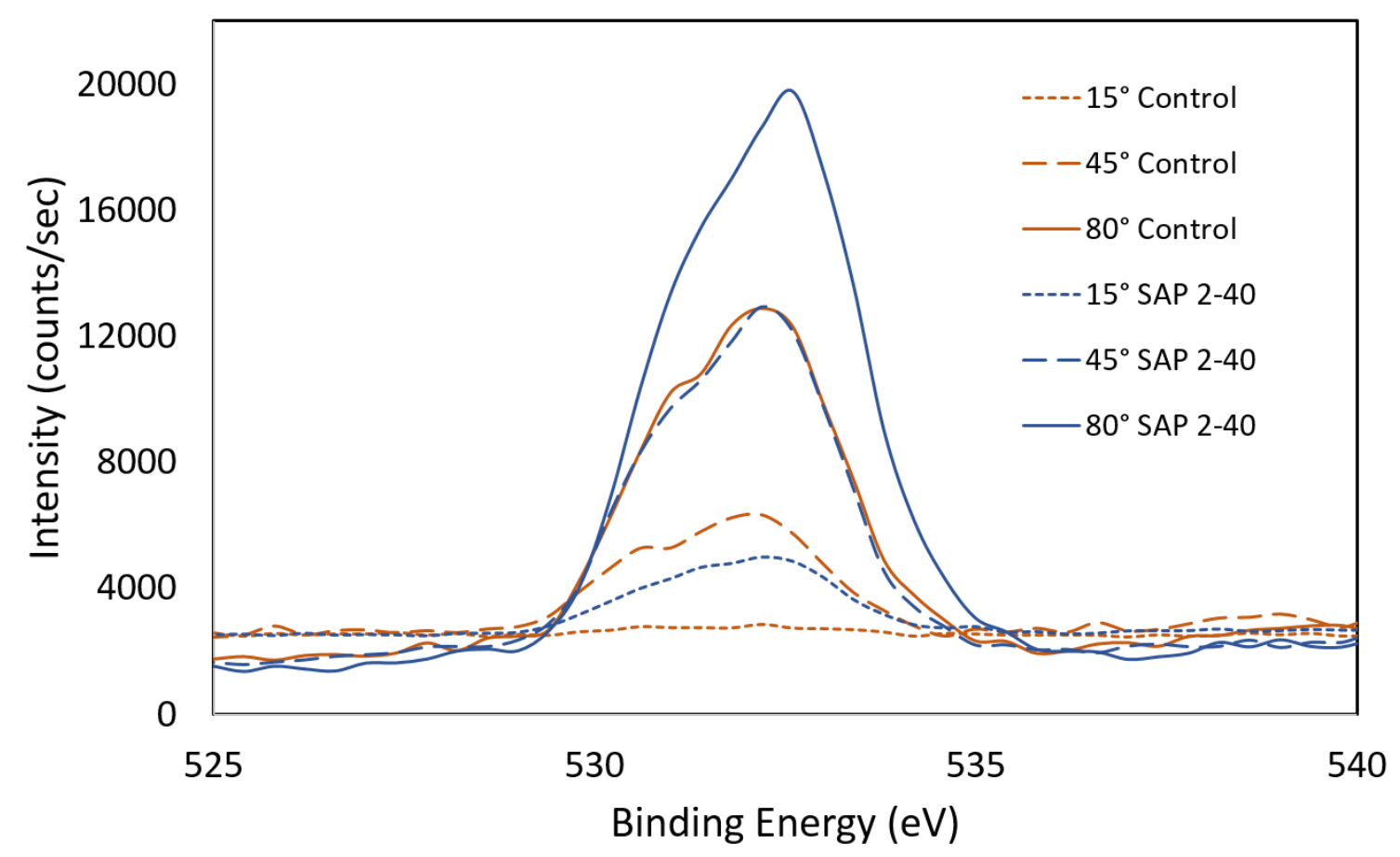

Figure 27. X-ray photoelectron spectra at the oxygen edge showing oxygen content at different detection depths in a control sample with no SAP and a sample with $2 \mathrm{wt} \%$ SAP 2-40. 


\subsection{Etch Process}

In order for DSA of BCPs to be considered successful for patterning applications, it is necessary to be able to selectively etch one of the BCP domains using existing etch processes. Due to the large presence of oxygen in PMeCAR, it is expected to have a higher etching rate than PS when using $\mathrm{O}_{2}$ reactive ion etching (RIE), and so the PMeCAR component of the BCP can be selectively removed [46]. Figure 28 shows SEM images of the steps throughout the etching process, along with representative cartoons of a PS-b-PMeCAR with 2 wt $\%$ SAP 2-40 thin film annealed at $170{ }^{\circ} \mathrm{C}$ for 5 minutes on a typical tri-layer stack on a silicon substrate [52]. The tri-layer stack was comprised of a $20 \mathrm{~nm}$ thick layer of $\alpha$-carbon, $5 \mathrm{~nm}$ of $\mathrm{SiN}_{\mathrm{x}}$, and approximately a $5 \mathrm{~nm}$ thick underlayer $(20 \% \mathrm{~S})$.

The thin film, before any etching, is shown in Figure 28a. The PMeCAR domain and the underlayer underneath were removed via etching using $\mathrm{O}_{2}$ plasma, shown in Figure 28b. The unremoved PS acted as an etch mask for the following etch step to remove the exposed $\mathrm{SiN}_{\mathrm{x}}$ layer using $\mathrm{CF}_{4} / \mathrm{CHF}_{3}$, as shown in Figure 28c. The final etch step used $\mathrm{O}_{2} / \mathrm{N}_{2}$ to remove the remaining PS layer and the $\alpha$-carbon, leaving the $\mathrm{SiN}_{\mathrm{x}}$ as an etch mask, shown in Figures $28 \mathrm{~d}$ and e, for pattern transfer into the silicon wafer. The cross-section SEM image, Figure 28e, shows the $\mathrm{SiNx}$ and $\alpha$-carbon layer. The features are approximately $10 \mathrm{~nm}$ wide, which was expected as the approximate half-pitch. These results show that PMeCAR can be selectively removed, and pattern transfer is successful throughout the etch process using fabrication compatible processing conditions. 
a)
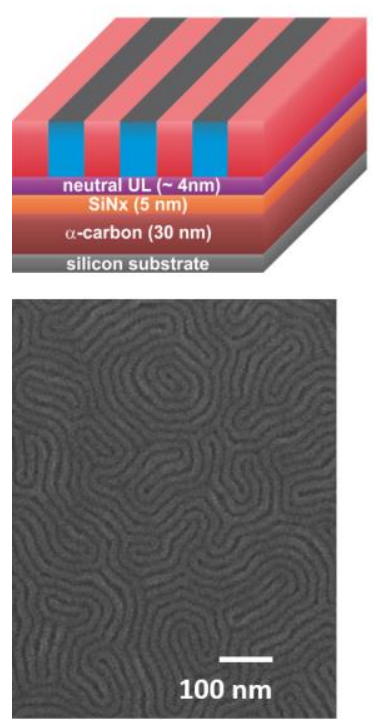

b)
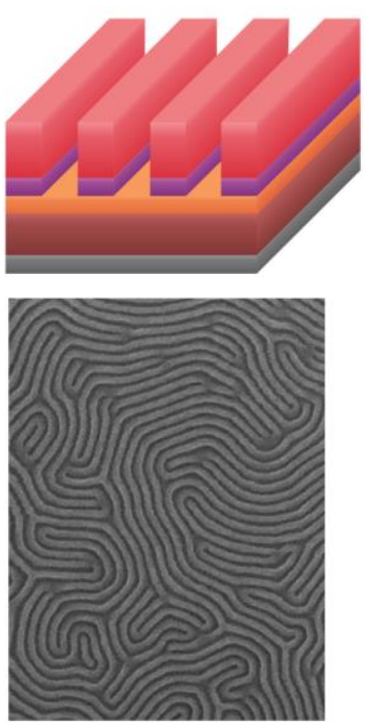

c)
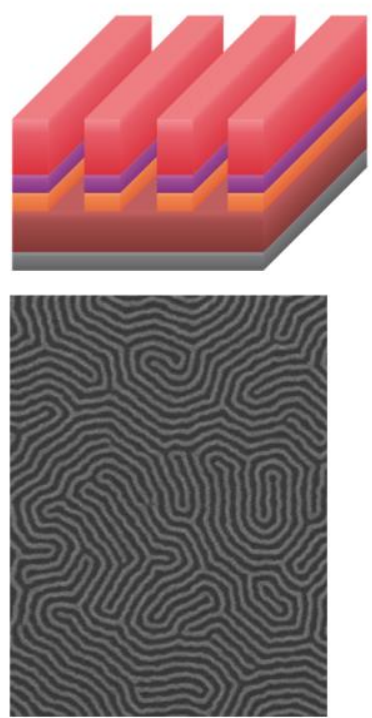

d)
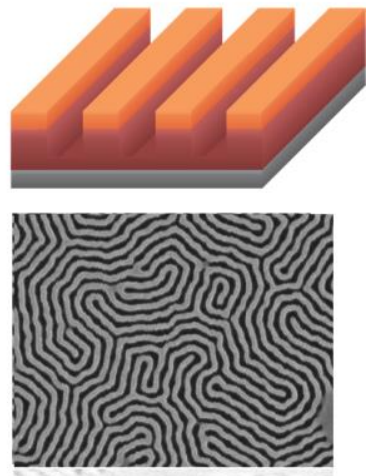

e)

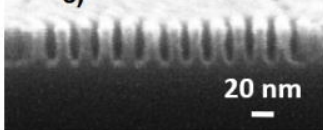

Figure 28. Scanning electron microscope images and corresponding cartoons of PS-bPMeCAR with 2 wt\% SAP 2-40 (a) before etching, (b) after $\mathrm{O}_{2}$ etch to remove PMeCar and the underlayer underneath it, (c) after $\mathrm{CF}_{4} / \mathrm{CHF}_{3}$ etch to remove $\mathrm{SiNx}$ layer, (d) topdown after $\mathrm{O}_{2} / \mathrm{N}_{2}$ etch to remove PS, remaining underlayer, and $\alpha$-carbon layer and, (e) a cross-section.

As an alternate to direct RIE etching of the thin film for pattern transfer, a PS-bPMeCAR with 2 wt \% SAP 2-40 film was modified using Sequential Infiltration Synthesis (SIS) with trimethyl aluminum (TMA). Sequential infiltration synthesis is a technique that uses inorganic materials to infiltrate the polymer film and form metal oxides through the use of gaseous precursors. The inorganic material and gaseous precursors are chosen to selectively react with only one of the $\mathrm{BCP}$ components. This is followed by a reaction with water vapor to provide growth sites for the inorganic material, in order to increase the etch resistivity of the selected domain. The metal oxide containing domain can then be used to facilitate pattern transfer. 
Previous literature mentioned that growth of aluminum oxide in the film relies on the chemical interaction between TMA and functional groups, such as carbonyls $(\mathrm{C}=\mathrm{O})$ [42]. The PS-b-PMeCAR BCP is an attractive candidate for using the SIS technique because of the carbonyl group present in PMeCAR. Using TMA and water vapor, aluminum oxide can grow in the bulk of the film. Using alternating cycles of TMA and water vapor, the TMA is expected to selectively interact with the carbonyl group present in PMeCAR, followed by a reaction with the water vapor to form aluminum oxide. Aluminum oxide is more etch resistant than either PS or PMeCAR, so its presence in PMeCAR makes the domain more etch resistive when using $\mathrm{O}_{2}$ RIE. Using SEM, the film was characterized pre-SIS process, post-SIS process, and post etch process, with the corresponding images shown in Figures 29a-c, respectively. Because aluminum is a heavier element than carbon or oxygen, aluminum backscatters more easily, and so appears brighter in an SEM image. The brighter lines seen in Figure 29b (post-SIS) in comparison to Figure 29a (pre-SIS) indicate the successful infiltration of aluminum into a selective domain. After etching the modified film with $\mathrm{O}_{2}$ plasma to remove the organic portion of the film, aluminum oxide was left, which could be used for further pattern transfer into the silicon substrate. This shows that SIS using TMA and water vapor is possible and is an available option to use with this $\mathrm{BCP}$ platform. 
a)
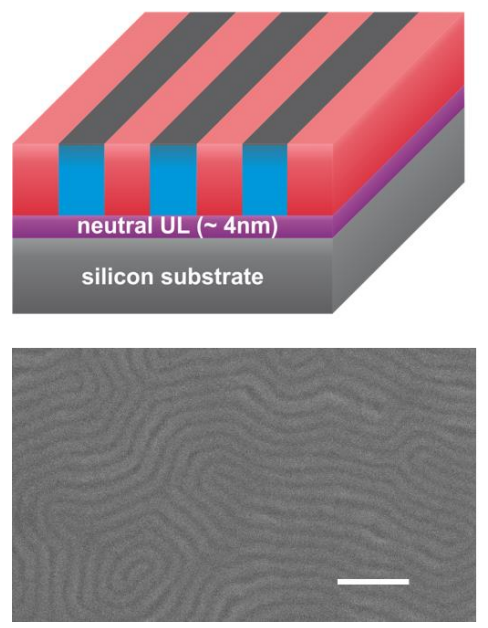

b)


c)


Figure 29. Scanning electron microscope images and corresponding cartoons of PS-bPMeCAR + 2 wt $\%$ SAP 2-40 a) before SIS process, b) post SIS process, and c) post $\mathrm{O}_{2}$ RIE etch removing PS domain. Scale bar: $100 \mathrm{~nm}$.

\subsection{Directed Self-Assembly using Graphoepitaxy}

In this section, DSA of PS-b-PMeCAR with a SAP additive was demonstrated to be successful via graphoepitaxy, using a polarity switched hardened photoresist-based pre-pattern. Figure 30a shows an SEM image of the pre-pattern. The pitch of the prepattern was approximately $200 \mathrm{~nm}$, and the trench width was approximately $80 \mathrm{~nm}$. The pitch of the $\mathrm{BCP}$ was approximately $20 \mathrm{~nm}$, and so the trench width had 4x multiplication ( $4 \mathrm{~L}_{0}$ width). The neutral underlayer was within the trenches of the pre-pattern, while the hardened sidewalls were polar, and so attracted the PMeCAR domain. Therefore, the PMeCAR domain was expected to align along the polar sidewalls, while the neutral underlayer and SAP additive enabled perpendicular orientation.

The SAP 2 series required a lower loading for uniform coverage of perpendicular lamellae in comparison to SAP 1. Furthermore, between SAP 2-40 and 2-80, less defects 
were seen on a thicker film using SAP 2-80. For these reasons, a formulation of PS-bPMeCAR with 2 wt \% SAP 2-80 was chosen to demonstrate the DSA process. The formulation was coated onto the pre-pattern and annealed at $170{ }^{\circ} \mathrm{C}$ for 5 minutes, the same conditions used in making all previous samples. The SEM image in Figure 30b shows some alignment of the BCP along the photoresist walls, but a large amount of defects.

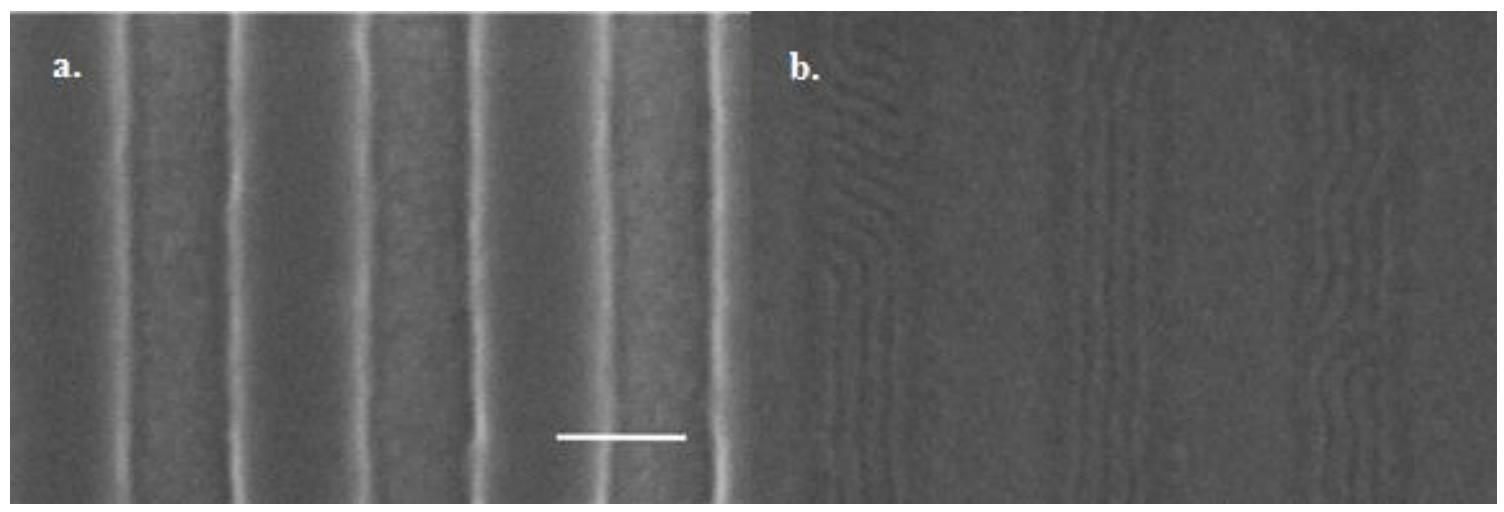

Figure 30. Scanning electron microscope images of (a) the graphoepitaxy pre-pattern and (b) alignment of PS-b-PMeCAR $+2 \mathrm{wt} \%$ SAP 2-80 when annealed at $170{ }^{\circ} \mathrm{C}$ for 5 minutes. Scale bar: $100 \mathrm{~nm}$.

Thermal annealing provides the mobility for microphase separation of the BCP to occur. As such, temperature $\left(170^{\circ} \mathrm{C}\right.$ and $\left.200{ }^{\circ} \mathrm{C}\right)$ and time $(5$ and 10 minutes $)$ were varied to allow for better mobility of the BCP in an attempt to reduce defects. Due to the large amount of defects formed with the initial annealing conditions used $\left(170{ }^{\circ} \mathrm{C}\right.$ for 5 minutes), a higher temperature and a longer anneal time were used to improve alignment [48]. Annealing conditions of temperature $200{ }^{\circ} \mathrm{C}$ and time of 5 minutes were used, with the higher temperature expected to increase mobility, but still kept sufficiently low so that the $\mathrm{BCP}$ would not degrade, allowing for observations on the effect of temperature on the 
BCP alignment to be made. Figure $31 \mathrm{~b}$ shows how at these conditions, slightly better alignment was seen, but defects were still present. When annealing time was doubled from 5 to 10 minutes for both temperatures $\left(170\right.$ and $\left.200{ }^{\circ} \mathrm{C}\right)$, much better alignment along the photoresist walls was achieved, as show in Figures 31c and 31d, respectively. Between annealing at 170 and $200{ }^{\circ} \mathrm{C}$ for 10 minutes, not much difference was seen in alignment. Based on these results, it appears that increase in time has a more significant effect in alignment improvement.

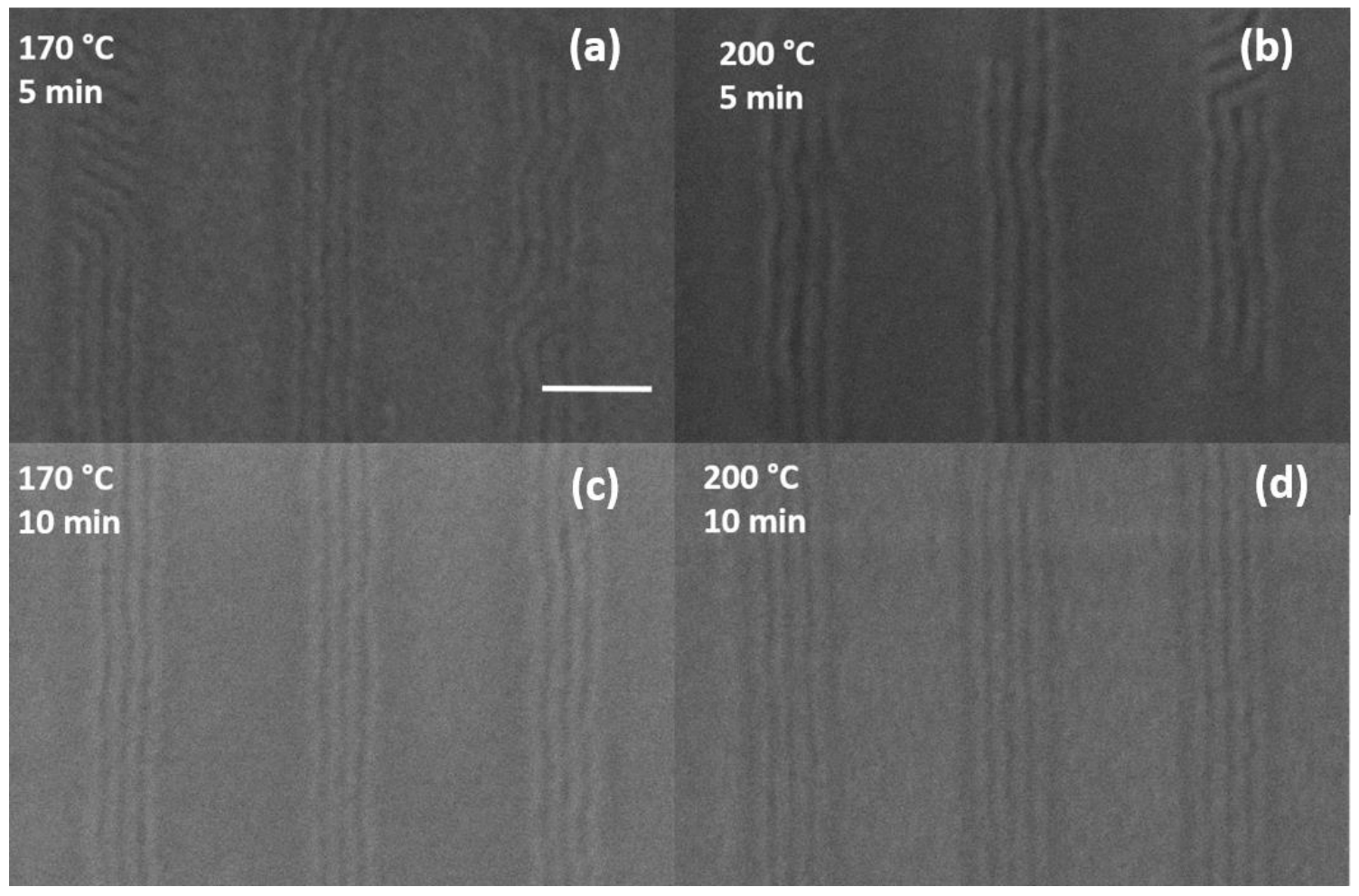

Figure 31. Scanning electron microscope images of PS-b-PMeCAR +2 wt $\%$ SAP 2-80 in pre-patterns annealed at (a) $170{ }^{\circ} \mathrm{C}$ for 5 minutes, (b) $200{ }^{\circ} \mathrm{C}$ for 5 minutes, (c) $170{ }^{\circ} \mathrm{C}$ for 10 minutes, and (d) $200{ }^{\circ} \mathrm{C}$ for 10 minutes. Scale bar: $100 \mathrm{~nm}$. 
To better determine the effect of time and temperature on alignment, a two factorial, two level, design of experiment was used to observe the main effects and interactions of the two parameters. A scale of one to four was used to qualitatively rate the alignment of the $\mathrm{BCP}$, with one representing bad alignment, and four representing good alignment. The corresponding annealing conditions for each SEM image in Figure 31 and the corresponding rating of the alignment are listed in Table 2.

Table 2. Design of experiment for time and temperature effect on DSA alignment

\begin{tabular}{|c|c|c|c|}
\hline Experiment & $\begin{array}{c}\text { Time } \\
\text { (minutes) }\end{array}$ & $\begin{array}{c}\text { Temperature } \\
\left({ }^{\circ} \mathrm{C}\right)\end{array}$ & Observation \\
\hline A & 5 & 170 & 1 \\
\hline B & 5 & 200 & 2 \\
\hline C & 10 & 170 & 4 \\
\hline D & 10 & 200 & 4 \\
\hline
\end{tabular}

Figure 32 shows the plots that represent the main effects of time and temperature on the alignment of the BCP. The positive slopes found on both plots show that both time and temperature individually improve alignment as their value is increased. Between the two parameters, the larger slope observed with an increase in time shows that time had a more significant effect than temperature in improving alignment. As for the interaction between the parameters, the plot is shown in Figure 33. At 5 minutes, an increase in temperature made a positive difference. At 10 minutes, with an increase in temperature, no change in the slope was seen, signifying that temperature did not make a difference in alignment in this case. Between the observations of the SEM and design of experiment results, it is concluded that at a lower anneal time, temperature plays a 
stronger role in alignment improvement. For a longer anneal time, temperature no longer contributes as significantly to alignment improvement.
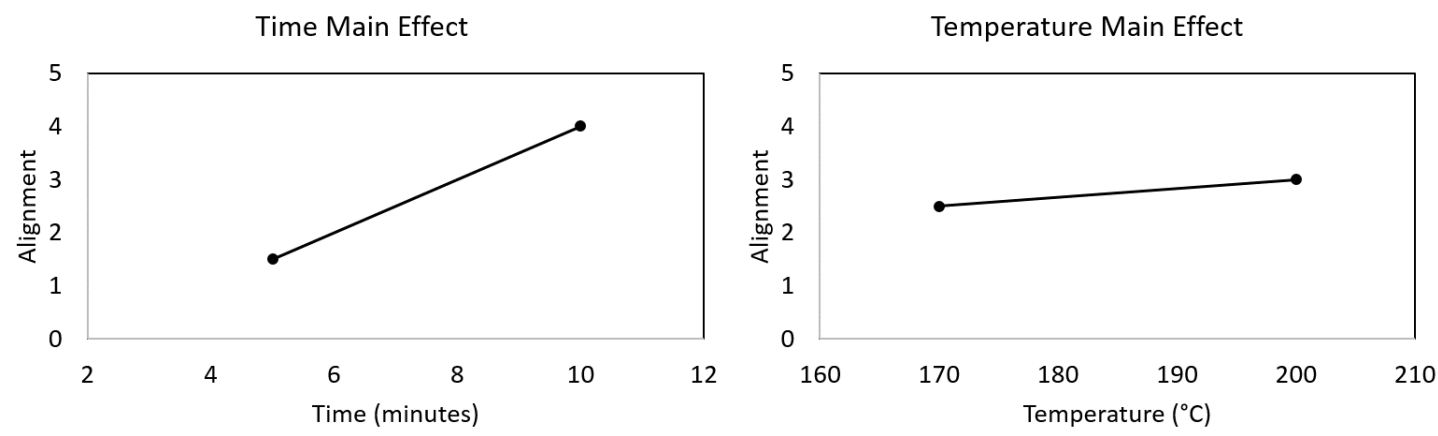

Figure 32. Design of experiment plots of a) the main effect of time on alignment improvement, and b) the main effect of temperature on alignment improvement.

\section{Parameter Interaction}

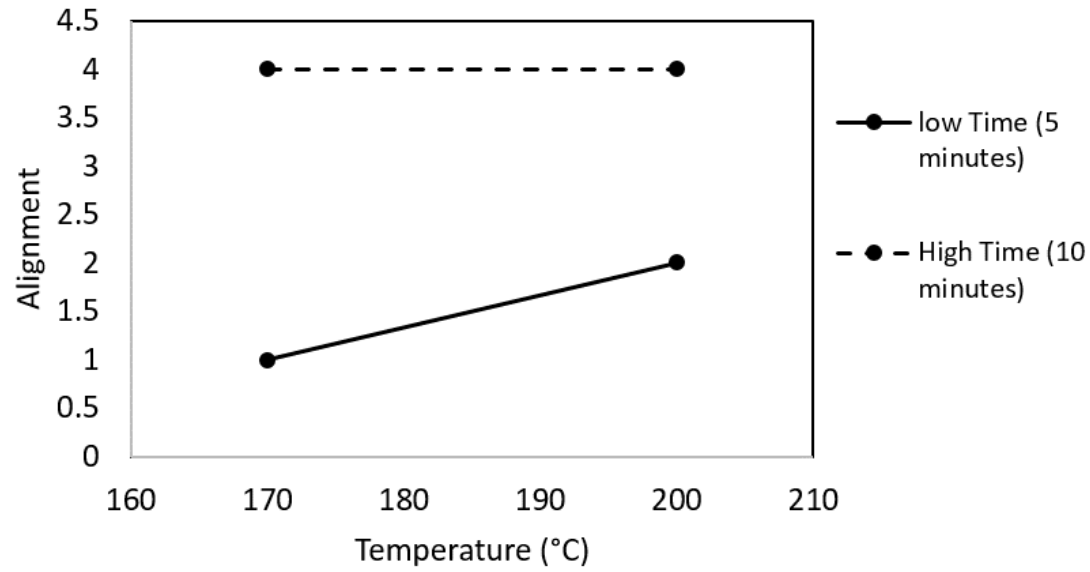

Figure 33. Design of experiment plot for time and temperature interaction on alignment improvement. 


\section{CHAPTER SIX}

\section{CONCLUSIONS}

A study of high- $\chi$ PS-b-PMeCAR BCP thin films with SAP additives for sub-20 nm pitch lamellae orientation control was reported. This method enables a fabrication process comparable to the current PS-b-PMMA process (i.e., spin coated from fabrication-friendly solvent PGMEA, thermally annealed, and no additional processing steps needed) for a BCP with sub-20 nm features that PS-b-PMMA does not offer. Using the method previously reported by Kim et al. to identify a neutral underlayer, PS-bPMeCAR on PS-r-PMMA with $20 \% \mathrm{~S}$ formed $0.5 \mathrm{~L}_{0}$ step height features [27]. With the addition of the SAP additive to the BCP formulation, successful orientation control at the BCP-air interface was demonstrated, as verified by AFM, GISAXS, and XPS.

Furthermore, low concentrations of 2-3 wt \% SAP were sufficient for obtaining uniform perpendicular lamellae in this formulation based method, as opposed to previous reported values of $10-20 \mathrm{wt} \%$.

The balance between the surface activity and concentration of the SAP was demonstrated to be significant in obtaining defect-free perpendicular lamellae. The SAP 1, which has a lower surface activity than either of the SAP 2 series compositions, required a higher concentration for uniform coverage. On the other hand, the more surface active SAP 2 series required a lower concentration for uniform coverage, as higher concentrations began forming parallel lamellae at the surface. Thickness studies on the SAP 2 series showed that for thicker films, a more surface active SAP is preferable 
for perpendicular lamellae to form, since the less surface active SAP (SAP 2-40) showed parallel lamellae formation in the thicker film.

Successful removal of PMeCAR from the pattern formed from a BCP with $2 \mathrm{wt}$ $\%$ SAP 2-40 formulation was demonstrated through the use of $\mathrm{O}_{2} \mathrm{RIE}$. Additionally, implementation of SIS to selectively incorporate aluminum oxide into the PMeCAR domain to decrease the etch rate in order to remove the PS domain through $\mathrm{O}_{2} \mathrm{RIE}$ was also successfully shown. Both methods gave similar results, as seen by SEM, demonstrating the potential use of this platform for pattern transfer applications.

Graphoepitaxy with $4 \mathrm{X}$ pitch density was successfully done using a BCP with 2 wt $\%$ SAP 2-80 formulation. The previous annealing conditions used $\left(170{ }^{\circ} \mathrm{C}, 5\right.$ minutes) showed defects when viewed with SEM, and so annealing temperature and time were modified to reduce defect formation. Design of experiment and SEM results showed higher temperatures have a positive effect on alignment at lower annealing times, while at longer annealing times, temperature no longer appeared to have much of an effect.

Overall, it was demonstrated that using a SAP based additive approach is promising for control orientation of high- $\chi$ BCPs, and is compatible with existing etch processes and graphoepitaxy schemes. Future work includes applying the SAP additives on other $\mathrm{BCP}$ platforms and morphologies to enable sub-20 $\mathrm{nm}$ pitch features for patterning work beyond lamellae morphology. Further optimization of the conditions for DSA alignment, beyond the demonstration of its compatibility shown here, would also be necessary for its successful use in patterning work. 


\section{REFERENCES}

1. R. A. Robison, "Moore's Law: Predictor and Driver of the Silicon Era, "World Neurosurg., 78, 399-403 (2012).

2. Intel (2015). Moore's Law 40th Anniversary [Online]. Available at http://www.intel.com/pressroom/kits/events/moores_law_40th. (accessed: 04-Sep2015). WWW Article.

3. D. of E. and C. E. of G. T. University (2015). Photolithography [Online]. Available at http://www.ece.gatech.edu/research/labs/vc/theory/photolith.html. (accessed: 10-Sep-2015). WWW Article.

4. J. I. Martín, J. Nogués, K. Liu, J. L. Vicent, and I. K. Schuller, “Ordered magnetic nanostructures: fabrication and properties, ” J. Magn. Magn. Mater., 256, 449$501(2003)$.

5. M. A. Morris, "Directed self-assembly of block copolymers for nanocircuitry fabrication," Microelectron. Eng., 132, 207-217 (2015).

6. C. M. Bates, M. J. Maher, D. W. Janes, C. J. Ellison, and C. G. Willson, "Block copolymer lithography, " Macromolecules, 47, 2-12 (2014).

7. T. P. Lodge, "Block Copolymers : Past Successes and Future Challenges," Macrolmol. Chem. Phys., 204, 265-273 (2003).

8. C. T. Black, R. Ruiz, G. Breyta, J. Cheng, M. Coulborn, K. Guarini, H. Kim, and Y. Zhang, "Polymer self-assembly in semiconductor microelectronics, ” IBM J. Res. Dev, 51, 605-633 (2007).

9. K. E. Sohn, "Surface Effects on the Structure of Block Copolymer and Homopolymer Thin Films, ” University of California, Santa Barbara (2008).

10. H. Kim, S.-M. Park, and W. D. Hinsberg, "Block Copolymer Based Nanostructures : Materials , Processes , and Applications to Electronics, ” Chem. Rev., 110, 146-177 (2010).

11. Y. Mai, F. Zhng, and X. Feng, "Polymer-directed synthesis of metal oxidecontaining nanomaterials for electrochemical energy storage, ” Nanoscale, 6, 106121 (2014).

12. D. Walton, G. Kellogg, and A. Mayes, “A free energy model for confined diblock copolymers,” Macromolecules, 27, 6225-6228 (1994).

13. C. M. Bates, T. Seshimo, M. J. Maher, W. J. Durand, J. D. Cushen, L. M. Dean, G. Blachut, C. J. Ellison, and C. G. Willson, "Polarity-switching top coats enable orientation of sub-10-nm block copolymer domains, " Science., 338, 775-779 (2012). 
14. H. Yoshida, H. S. Suh, a Ramirez-Hernandez, J. I. Lee, K. Aida, L. Wan, Y. Ishida, Y. Tada, R. Ruiz, J. de Pablo, and P. F. Nealey, "Topcoat Approaches for Directed Self-Assembly of Strongly Segregating Block Copolymer Thin Films, ” J. Photopolym. Sci. Technol., 26, 55-58 (2013).

15. S. Kim, P. F. Nealey, and F. S. Bates, "Decoupling bulk thermodynamics and wetting characteristics of block copolymer thin films, " ACS Macro Lett., 1, 11-14 (2012).

16. A. Vora, IBM Research- Almaden, private communication (November 2015).

17. S. J. Jeong, J. Y. Kim, B. H. Kim, H. S. Moon, and S. O. Kim, “Directed selfassembly of block copolymers for next generation nanolithography, ” Mater. Today, 16, 468-476 (2013).

18. S. O. Kim, H. H. Solak, M. P. Stoykovich, N. J. Ferrier, J. J. De Pablo, and P. F. Nealey, "Epitaxial self-assembly of block copolymers on lithographically defined nanopatterned substrates., " Nature, 424, 411-4 (2003).

19. R. A. Segalman, H. Yokoyama, and E. J. Kramer, "Graphoepitaxy of spherical domain block copolymer films, ” Adv. Mater., 13, 1152-1155 (2001).

20. G. S. Doerk, J. Y. Cheng, G. Singh, C. T. Rettner, J. W. Pitera, S. Balakrishnan, N. Arellano, and D. P. Sanders, "Enabling complex nanoscale pattern customization using directed self-assembly,” Nat. Commun., 5, 1-8 (2014).

21. H. Tsai, J. W. Pitera, H. Miyazoe, S. Bangsaruntip, S. U. Engelmann, C. Liu, J. Y. Cheng, J. J. Bucchignano, D. P. Klaus, E. A. Joseph, D. P. Sanders, M. E. Colburn, and M. A. Guillorn, "Two-Dimensional Pattern Formation Using Graphoepitaxy of PS - b - PMMA Block Copolymers for Advanced FinFET Device and Circuit Fabrication," ACS Nano, 8, 5227-5232 (2014).

22. P. Mansky, P. Haikin, and E. L. Thomas, "Monolayer films of diblock copolymer microdomains for nanolithographic applications, ” J. Mater. Sci., 30, 1987-1992 (1995).

23. G. J. Kellogg, D. G. Walton, A. M. Mayes, P. Lambooy, T. P. Russell, P. D. Gallagher, and S. K. Satija, "Observed Surface Energy Effects in Confined Diblock Copolymers,” Phys. Rev. Lett., 76, 2503-2506 (1996).

24. P. Mansky, Y. Liu, E. Huang, T. P. Russell, and C. J. Hawker, "Controlling polymer-surface interactions with random copolymer brushes, ”Science, 275, 1458-1460 (1997).

25. P. Mansky, T. P. Russell, C. J. Hawker, M. Pitsikalis, and J. Mays, “Ordered diblock copolymer films on random copolymer brushes, ” Macromolecules, $\mathbf{3 0}$, 6810-6813 (1997).

26. R. D. Peters, X. M. Yang, T. K. Kim, B. H. Sohn, and P. F. Nealey, "Using Self- 
Assembled Monolayers Exposed to X-rays To Control the Wetting Behavior of Thin Films of Diblock Copolymers, ” Langmuir, 16, 4625-4631 (2000).

27. S. Kim, C. M. Bates, A. Thio, J. D. Cushen, C. J. Ellison, C. G. Willson, and F. S. Bates, "Consequences of surface neutralization in diblock copolymer thin films," ACS Nano, 7, 9905-9919 (2013).

28. P. Mansky, T. Russell, C. Hawker, J. Mays, D. Cook, and S. Satija, “Interfacial Segregation in Disordered Block Copolymers: Effect of Tunable Surface Potentials," Phys. Rev. Lett., 79, 237-240 (1997).

29. J. Zhang, M. B. Clark, C. Wu, M. Li, P. Trefonas, and P. D. Hustad, “Orientation Control in Thin Films of a High- $\chi$ Block Copolymer with a Surface Active Embedded Neutral Layer., ” Nano Lett., 16, 728-35 (2016).

30. J. G. Son, K. W. Gotrik, and C. A. Ross, "High-aspect-ratio perpendicular orientation of PS-B -PDMS thin films under solvent annealing," ACS Macro Lett., 1, 1279-1284 (2012).

31. S. H. Kim, M. J. Misner, T. Xu, M. Kimura, and T. P. Russell, "Highly oriented and ordered arrays from block copolymers via solvent evaporation," Adv. Mater., 16, 226-231 (2004).

32. A. Vora, R. J. Wojtecki, K. Schmidt, A. Chunder, J. Y. Cheng, A. Nelson, and D. P. Sanders, "Development of polycarbonate-containing block copolymers for thin film self-assembly applications,” Polym. Chem., 7, 940-950 (2016).

33. E. Han, K. O. Stuen, M. Leolukman, C. C. Liu, P. F. Nealey, and P. Gopalan, "Perpendicular orientation of domains in cylinder-forming block copolymer thick films by controlled interfacial interactions, ” Macromolecules, 42, 4896-4901 (2009).

34. J. N. L. Albert, W. S. Young, R. L. Lewis, T. D. Bogart, J. R. Smith, and T. H. Epps, "Systematic study on the effect of solvent removal rate on the morphology of solvent vapor annealed ABA triblock copolymer thin films, ” ACS Nano, 6, 459466 (2012).

35. C. Sinturel, M. Morris, and M. Hillmyer, "Solvent Vapor Annealing of Block Polymer Thin Films,” Macromolecules, 46, 5399-5415 (2013).

36. R. J. Albalak, M. S. Capel, and E. L. Thomas, "Solvent swelling of roll-cast triblock copolymer films, ” Polymer., 39, 1647-1656 (1998).

37. X. Zhang, K. D. Harris, N. L. Y. Wu, J. N. Murphy, and J. M. Buriak, "Fast Assembly of Ordered Block Copolymer Nanostructures through Microwave Annealing, ” ACS Nano, 4, 7021-7029 (2010).

38. E. Huang, T. P. Russell, C. Harrison, P. M. Chaikin, R. A. Register, C. J. Hawker, and J. Mays, "Using Surface Active Random Copolymers To Control the Domain 
Orientation in Diblock Copolymer Thin Films," Macromolecules, 31, 7641-7650 (1998).

39. I. Keen, A. Yu, H. Cheng, K. S. Jack, T. M. Nicholson, A. K. Whittaker, and I. Blakey, "Article pubs.acs.org/Langmuir Control of the Orientation of Symmetric Poly(styrene)-block- poly(D,L-lactide) Block Copolymers Using Statistical Copolymers of Dissimilar Composition," Langmuir, 45, 15876-15888 (2012).

40. H. Li, W. Gu, L. Li, Y. Zhang, T. P. Russell, and E. B. Coughlin, "Synthesis of semicrystallinelfluorinated side-chain crystalline block copolymers and their bulk and thin film nanoordering, " Macromolecules, 46, 3737-3745 (2013).

41. D. K. Owens and R. C. Wendt, "Estimation of the surface free energy of polymers, ” J. Appl. Polym. Sci., 13, 1741-1747 (1969).

42. M. Biswas, J. A. Libera, S. B. Darling, and J. W. Elam, "Kinetics for the Sequential Infiltration Synthesis of Alumina in Poly(methyl methacrylate): An Infrared Spectroscopic Study,” J. Phys. Chem. C, 119, 14585-14592 (2015).

43. R. A. Farrell, N. Petkov, M. T. Shaw, V. Djara, J. D. Holmes, and M. A. Morris, "Monitoring PMMA Elimination by Reactive Ion Etching from a Lamellar PS-bPMMA Thin Film by ex Situ TEM Methods," Macromolecules, 43, 8651-8655 (2010).

44. J. W. Elam, M. Biswas, S. Darling, A. Yanguas-Gil, J. D. Emery, A. B. F. Martinson, P. F. Nealey, T. Segal-Peretz, Q. Peng, J. Winterstein, J. A. Liddle, and Y.-C. Tseng, "New Insights into Sequential Infiltration Synthesis, " ECS Trans., 69, 147-157 (2015).

45. Y. C. Tseng, Q. Peng, L. E. Ocola, J. W. Elam, and S. B. Darling, "Enhanced block copolymer lithography using sequential infiltration synthesis, ”J. Phys. Chem. C, 115, 17725-17729 (2011).

46. H. Gokan, S. Esho, and Y. Ohnishi, "Dry Etch Resistance of Organic Materials," J. Electrochem. Soc., 130, 143-146 (1983).

47. O. Coulembier, D. P. Sanders, A. Nelson, A. N. Hollenbeck, H. W. Horn, J. E. Rice, M. Fujiwara, P. Dubois, and J. L. Hedrick, "Hydrogen-bonding catalysts based on fluorinated alcohol derivatives for living polymerization," Angew. Chemie - Int. Ed., 48, 5170-5173 (2009).

48. A. M. Welander, H. Kang, K. O. Stuen, H. H. Solak, M. Müller, J. J. De Pablo, and P. F. Nealey, "Rapid Directed Assembly of Block Copolymer Films at Elevated Temperatures," Macromolecules, 41, 2759-2761 (2008).

49. A. Hexemer, W. Bras, J. Glossinger, E. Schaible, E. Gann, R. Kirian, A. MacDowell, M. Church, B. Rude, and H. Padmore, "A SAXS/WAXS/GISAXS Beamline with Multilayer Monocromator,” J. Phys. Conf., 247, 012007 (2010). 
50. J. Ilavsky and P. Jemian, "Irena: tool suite for modeling and analysis of smallangle scattering," J. Appl. Crystallogr., 42, 347-353 (2009).

51. P. Busch, D. Posselt, D.-M. Smilgies, M. Rauscher, and C. M. Papadakis, "Inner Structure of Thin Films of Lamellar Poly(styrene-b-butadiene) Diblock Copolymers As Revealed by Grazing-Incidence Small-Angle Scattering," Macromolecules, 40, 630-640 (2007).

52. M. Weimer, Y. Wang, C. J. Neef, J. Claypool, K. Edwards, and Z. Zhu, "Materials for and performance of multilayer lithography schemes," Procs. SPIE Conf., 2007. 